\title{
Measuring Permeabilities of Middle-Bakken Samples Using Three Different
}

\section{Methods}

Jun He and Kegang Ling, University of North Dakota

Corresponding Author: Kegang Ling

Phone: 701-777-3194

Fax: 701-777-1820

Email: kegang.ling@engr.und.edu

Keywords: Permeability Measuremnt, Bakken Formation, Tight Rock

\begin{abstract}
In recent years, petroleum exploration and production from the Williston Basin at the Bakken Formation has garnered great success. Producing hydrocarbons from the Bakken Formation is challenging due to its low porosity and permeability. Investigating the permeability of the Bakken Formation is required in order to better understand the performance of wells that produce hydrocarbons. In addition, permeability is one of the key parameters in modeling fluids flow in reservoir simulation matrices. Unfortunately, the measurement of permeability of tight rocks, such as in Bakken samples, is time consuming and expensive due to their low to extremely low permeability; in addition, sometimes the results from different methods are not in good agreement.
\end{abstract}

Because of the high uncertainty in measuring the permeability of tight rock, it is worthwhile to investigate permeability through different methods in order to reduce uncertainty. In this study, we measured the permeability of tight rocks utilizing three different methods with the same setup. The investigated methods were the oscillating pulse method, the downstream pressure buildup method, and the radius-of-investigation method. In this way, the 
comparison provides uncertainty and magnitude and indicates the possible presence of heterogeneities and lamination.

\section{Introduction}

Hydrocarbon production from the Bakken formation has gained great success at the Williston Basin in recent years through the application of horizontal well and hydraulic fracturing technologies. Because permeability plays an important role in reservoir modeling and production forecast, it is imperative to investigate permeability of the Bakken formation in order to gain a better understanding of reservoir performance.

Based on the experimental work from Darcy (1856), many methods have been presented to improve the accuracy and efficiency of measurement. These methods, based on flow regime, can be classified into two categories: steadystate flow methods and unsteady-state flow methods. Steady-state flow methods measure permeability under steadystate conditions. Aside from low flow rates across the core plug being difficult to measure and control, these tests are quite time consuming. In this case, unsteady state flow is applied to estimate permeability. Brace et al. (1968) introduced a transient flow method to measure the permeability of Westerly granite. From this, many unsteady-state methods have been proposed to measure the permeability of tight rocks. Most of these methods fall into three categories: the pulse decay method, the Gas Research Institute (GRI) method, and the oscillating pulse method.

For the pulse-decay method, the sample has both an upstream reservoir and a downstream reservoir. A pressure pulse, which is applied at the upstream reservoir, will decay over time. The permeability is estimated by analyzing the decay characteristics of the pressure pulse (Brace et al., 1968). Dicker and Smits (1988) improved the pressure pulse-decay method by showing a general solution of the differential equation which describes the pressure decay curve. Based on this solution, they theoretically pointed out that fast and accurate measurements are possible when the volumes of the upstream and downstream reservoirs in the equipment are equal to the pore volume of the sample. Jones (1997) pointed out that the initial pressure equilibration step is the most time-consuming part of the pulse-decay technique. To avoid the equilibrium state, Jones' method utilizes a smooth pressure gradient, which requires smaller upstream and downstream reservoirs. To account for adsorption during pulse-decay measurement, Cui et al. (2009) presented their method which can describe gas transport in low permeability reservoir more reliably and accurately. Metwally (2011) proposed another pulse-decay method by keeping the upstream reservoir pressure 
constant leading to an infinitely large volume of the upstream reservoir, so that the ratio of upstream reservoir volume to downstream reservoir volume is infinite. Thus, the solution of the pulse-decay measurements can be simplified.

The GRI method differs from the pulse-decay method in that the measurement is carried out on crushed rock samples; a pressure pulse is applies on unconfined crushed rock particles. Permeability is then obtained through the analysis of the pressure decay over time. Cui et al. (2009) developed a late-time method utilizing data from either pulse-decay or GRI experiment to determine the permeability. The GRI method has the advantage of a shorter experimental time as compared with other methods. Unfortunately, permeability measured from crushed samples can differ by 2 to 3 orders of magnitude among different commercial laboratories (Passey et al., 2010 and Tinni et al., 2012). Another limitation of this method is that the microcracks in the crushed particles essentially violate the GRI assumptions. This leads to an overestimate of permeability (Tinni et al., 2012). To improve the accuracy and consistency of the GRI method, Sinha (2012) developed cylindrical calibration standards based on first principles to calibrate the low permeability measurement apparatus. The GRI method is not used in this study because of large permeability differences between crushed and intact samples.

The oscillating pulse method estimates rock permeability by interpreting amplitude attenuation and phase retardation in the sinusoidal oscillation of the pore pressure as a pressure pulse propagates through a sample. At the beginning of the experiment, the sample pore pressure, the upstream reservoir pressure, and the downstream reservoir pressure are stabilized. Then a pressure wave is generated in the upstream reservoir and propagates through a core plug. The permeability can be obtained by using the information of the amplitude attenuation and phase shift between the upstream reservoir pressure wave and the derived downstream reservoir pressure wave at the downstream side of the sample. Although this method can measure the permeability in a relatively short time without destroying the sample, as the GRI method does, the accuracy of permeability obtained from this method relies on the signal-to-noise ratio and data analysis techniques (Kranz et al., 1990).

Normally, permeabilities measured by the different methods are not in good agreement. Bertoncello (2013) concluded that the steady-state method with critical fluid provides much more consistent and acceptable results after comparing permeability measurements performed at several commercial and research laboratories using four different techniques. However, Bertoncello (2013) did not mention the time of measurement for each method. In 
fact, the measurement of tight rock permeability, such as in Bakken samples, is time consuming and expensive due to their low permeabilities. In addition, the results given by Lab_1 from transient methods are consistent and acceptable, and Lab_1 is the only laboratory which provides different methods.

In this study, we introduced a testing process to measure the permeabilities of tight rocks with three different methods under the same procedure. These methods are the oscillating pulse method, the downstream pressure buildup method, and the radius-of-investigation method. In this way, not only the comparabilities of the results from these three methods increased, but the difference among the results is also useful for indicating the heterogeneity and/or microcracks of the rock. The theory, development of governing equations, and derivation of solutions for the downstream pressure buildup method and the radius-of-investigation method are shown in Appendices B and C. The experiment procedures and data analyses of these two methods are shown in "Methodology" and "Results and Discussions".

\section{Methodology}

\section{Sampler and Equipment}

The Bakken Formation, which formed in the Williston Basin with an offshore marine environment, consists of three members: the upper shale, the lower shale, and the lithologically variable middle member. In this study the siltstone Middle Bakken core samples from one well in North Dakota were chosen to represent the tight rocks. The core plugs are cylindrical with dimensions of one inch in diameter and two inches in length.

First, the cylindrical core plug was covered with copper sheeting in order to both form a gas-tight seal on the cylindrical wall of the sample and to apply radial confining pressure. Then the core plug was mounted in a sample holder with flexible rubber sleeves at both ends of the plug (Figure 1). Finally, the sample holder was put into a vessel flooded with mineral oil, in which the sample could be hydrostatically compressed by hydraulically applying force to the plug. To minimize the volume of the downstream reservoir, a small pocket was implemented inside the downstream end-cap (Figures 1 and 2). The volume of downstream reservoir was $0.63 \mathrm{cc}$.

The equipment used to perform the experiments was an AutoLab 1500, which is made by New England Research Inc. Figure 2 presents a conceptual diagram of the test facility. One temperature transducer (TT1) is used to measure the temperature. Four pressure transducers (PT1, PT2, PT3, and PT4) are used to measure the upstream reservoir 
pressure, the confining pressure at the flank of the core, the downstream reservoir pressure, and the axial pressure at the ends of the core, respectively.

\section{Method 1: Oscillating Pulse Measurement Method}

Figure 3 indicates the theory of the aforementioned oscillating pulse method (Kranz et al., 1990). A fixed amplitude, fixed-frequency oscillation in the upstream reservoir pressure results in a reduced amplitude and phase-shifted pressure oscillation in the downstream reservoir after diffusing through the core sample. The amplitude ratio and phase shift provide information about the hydraulic properties of the rock. Based on these pressure responses, an analytical solution for permeability can be calculated from either the amplitude ratio or the phase shift. The relationship between the upstream and downstream perturbations is a function of the length, cross-sectional area, permeability, specific storage of the sample, the viscosity of the fluid, and the compressibility of the fluid. Appendix A provides the derivation of equations for this method given by Kranz et al. (1990). However, a strong dependence

on the ratio of permeability to specific-storage creates a situation where an error in the determination of one parameter (i.e. specific storage) will lead to an error in the determination of the other (i.e. permeability).

Permeability was measured using the oscillating method with the AutoLab 1500 system (Boitnott 1997), which performs sensitivity studies to provide a means of computing errors associated with the insensitivity of the solution to the separation of permeability and specific storage. However, the data analysis technique is not shared by New England Research Inc.

To validate the permeability from the oscillating method, we used two other methods: the downstream pressure buildup (Jones 1997) and a new method, called the radius-of-investigation method, developed in this study, which is based on the solutions to the diffusivity equation. Both methods can reduce testing time. One stops at point "A"; the other stops at point "B" (Figure 5).

\section{Introduction of Diffusivity Equation}

The estimations of permeability by the downstream pressure buildup method and the radius-of-investigation method require the solution of the diffusivity equation for the Darcy flow through the core sample. To derive the diffusivity equation, the following assumptions are made: 1) the core is homogeneous, 2) the properties of the rock are 
constant, 3) the flow in the cylindrical core is laminar, and 4) the flow in the core is isothermal. Because the permeability of tight rock is low, nitrogen gas is used as the test fluid in our experiment. The gas flows from the left-side of the core, through the core, and out of the right-side of the core as shown in Figure 4.

Considering a control volume (from $x$ to $x+\Delta x$ ), which is the volume that the gas flows in from $x$ and out at $x+\Delta x$ during a certain time period $\Delta t$, and combining the mass conservation, Darcy's law, real gas law, and the gas pseudo-pressure concept (Al-Hussainy, 1966), a diffusivity equation for linear gas flow is stated as:

$$
\frac{\partial^{2}}{\partial \mathrm{x}^{2}}[\mathrm{~m}(p)]=\frac{\varphi \mu c_{t}}{k} \frac{\partial}{\partial t}[\mathrm{~m}(p)]
$$

where $m(p)$ is the gas pseudo-pressure, which is expressed as

$$
\mathrm{m}(p)=\int_{\mathrm{p}_{\mathrm{b}}}^{\mathrm{p}} \frac{2 p}{\mu z} \mathrm{dp}
$$

\section{Method 2: Downstream Pressure Buildup Measurement Method}

In the downstream pressure buildup method, the upstream reservoir pressure is kept constant throughout the entire test and the pressure buildup is observed in the downstream reservoir when the gas flows into it. The section "BA" in Figure 5 shows the pressure change in the downstream reservoir as a function of time.

To calculate the permeability from the buildup curve of the measured downstream reservoir pressure, the solution to the diffusivity Equation (1) needs to be known. Permeability is then estimated through Equation (B-16). The derivation of equations for this method is in Appendix B.

\section{Method 3: Radius-of-Investigation Measurement Method}

Based on the Radius-of-Investigation Concept (Lee, 1982), a new method was proposed to measure core permeability. When doing the permeability test using the downstream pressure buildup method, it was observed that the downstream reservoir pressure did not increase immediately when the upstream reservoir was connected with the core plug. The lower the permeability, the longer the delay time was observed. The time that a pressure disturbance 
propagates through a core sample is a function of the permeability of the rock. Therefore, the low to extremely low permeability of Bakken samples can be calculated by measuring the delaying time, which is the time that the pressure disturbance propagates from the upstream end of the core plug to the downstream end of the core plug.

The pressure disturbance concept is applied here to estimate the propagation of pressure in the core plug. First, a pressure disturbance was introduced by either increasing the upstream reservoir pressure or decreasing the downstream reservoir pressure instantaneously; then the time $t_{m}$ at which the disturbance at location $x$ reaches its maximum was determined. With measured $t_{m}$ and given core geometry, the permeability can be obtained using Equation (C-7). The derivation of equations for this method is in Appendix C.

\section{Measurement Procedure}

The determination of the permeability is a three-step process for all of these three methods, namely installing the core plug into the AutoLab 1500, running the test, and analyzing the resultant data.

1) Installing the core plug into the AutoLab 1500

First, the sample is placed into the vessel; then the vessel is filled with mineral oil and the confining pressure is increased to the desired level $\left(p_{c}\right)$. The valve between the core plug and the upstream reservoir is closed. Dry nitrogen is used to fill the upstream reservoir, and the upstream reservoir pressure is increased to the desired level $\left(p_{l}\right)$. The downstream reservoir is at atmospheric pressure. Notice that the confining pressure must be greater than the upstream reservoir pressure.

\section{2) Running the test}

The start time is recorded when the valve between the core plug and the upstream reservoir is opened. During the entire test, the upstream reservoir and confining pressures are constant. The pressures are monitored and recorded at both the upstream and downstream ends of the sample.

Figure 5 shows the change of downstream reservoir pressure during the test. A constant pressure is applied at the upstream end of the core plug, and the pressure at the downstream end of the core plug is built up. For the radius-ofinvestigation method, the test ends when the downstream reservoir pressure starts to increase, which is at point " $\mathrm{B}$ " in Figure 5. For the downstream pressure buildup method, the test ends when the downstream reservoir pressure is 
equal to the upstream reservoir pressure, which is at point "A" in Figure 5. For the oscillating pulse method, the test ends at the point "C" in Figure 5.

\section{3) Analyzing the resultant data}

For the radius-of-investigation method, after finding the time of point "B", the permeability can be obtained using Equation (C-7) or (C-8). To better determine point "B", the section from constant downstream pressure to downstream pressure buildup is amplified. Then the beginning point of the increasing in downstream pressure is selected as point "B" (Figure 5). For the oscillating pulse method, the AutoLab 1500 system directly gives the permeability. To obtain the permeability using the downstream pressure buildup method, first the pressure difference is calculated using a logarithm scale form equation, $\left.\ln [\Delta p(t)]=\ln \mid p_{1}{ }^{2}(0)-p_{2}{ }^{2}(t)\right\rfloor$. Then, from the plot (Figure B-1), we obtain the slope $s$. Finally, Equation (B-16) is used to obtain the permeability of the rock (see Appendix B).

\section{Results and Discussions}

The permeabilities of six core plugs were measured using the oscillating pulse method, the downstream pressure buildup method, and the radius-of-investigation method. The pressure-time graphs are shown in Figure 5, Figure B1, and Figures D-1 through D-10. The parameters that were used in the tests and experiment results are shown in Table 1. The permeabilities from the downstream pressure buildup method and the radius-of-investigation method are close to those from the oscillating pulse method (Figure 6). Therefore, the downstream pressure buildup method and the radius-of-investigation method provide the same order of magnitude of permeability as the oscillating pulse method for tight rocks. This indicates that the uncertainty has been reduced materially. Figure 5 shows that the measured time of the radius-of-investigation method (ends at point "B") requires less time than the oscillating pulse method (ends at point "C").

The pressure buildup method, which is based on the pulse-decay method, is the transformation of a mature technique to measure the low permeability. Our study managed to reduce the downstream reservoir volume as much as possible in order to reduce the operation time. It requires less time than the oscillating pulse method and is a faster alternative to the steady-state, commonly accepted method. It is worth mentioning that to reduce the uncertainty during this procedure, the data that is chosen to calculate the slope $s$ should be part of the data between point "B" 
and "A" with high signal-to-noise ratio (Figure 5). Because selecting the whole curve to fit will increase slope $s$, or using the earlier data to fit will significantly reduce $s$ (Figure B-1), middle data with high signal-to-noise ratio should be selected (Figure 7). Doing so ensures the consistency of the calculation of the slope $s$ and reduces the uncertainty in the obtained permeability. For these tests, the permeability range of Core \#1 (Figure 7) was checked after calculating the compressibility and viscosity of the gas as response variables depending on the pore pressure. The data in measurement time from $1500 \mathrm{~s}$ to $3500 \mathrm{~s}$ give a reliable permeability because of high signal-to-noise ratio. Permeability in this time interval ranges from 0.1 to $0.12 \mu \mathrm{D}$. Therefore, the average value of permeability is acceptable.

The radius-of-investigation method requires the least amount of the time to perform and results in reliable data. It utilizes the propagation speed of the pressure wave in a certain media to calculate the permeability. This method provided a fast measurement of the low permeability. The measurement time for this method is 10 times less than the downstream pressure buildup method in this study. The radius-of-investigation method not only can be used to measure low permeability, but also can be used to measure high permeability by replacing the gas fluid with a liquid fluid. Other than the human introduced random error, the major uncertainty source in this method is mainly from the selection of point "B". To reduce the uncertainty, the beginning of the responding time (or point "B") is manually selected. It is easy to identify the starting point of downstream pressure buildup, which is point "B", through amplifying the short time interval as shown in Figure 5.

After we compared our results with those of Bertoncello's investigation (2013), the permabilities from these three methods are close. This increases the confidence level. Therefore, the comparative measurement and analysis has value by providing fast and inexpensive measurements and can help quantify uncertainties. In theory, the pore pressure differences in the three methods can cause the permeability difference because high pore pressure leads to a larger pore volume and thus a higher permeability. However, the results show that the lowest permeability is determined by oscillating pulse method, which is under the highest pore pressure. The reason is that the oscillating pulse method is proffered by AutoLab 1500 system which considers the storage of the sample; this implies that the porosity of the samples is a variable in this method, and the porosity decreases with the effective pressure increase. However, for the other two methods, in this study we assume that the value of porosity is constant. It is equal to the 
one measured at $1 \mathrm{~atm}$. In fact with effective pressure increase, porosity will be reduced; thus, with a large porosity value, the permeability calculated by the other two methods is greater than the actual permeability.

If the results from these three methods are not close, it will not always be the case that the confidence level is low. It may indicate that the rock has a nonuniform pore structure. The heterogeneity of rock and/or microcracks may be the main causes. Considering the high pore pressure in the oscillating pulse and downstream pressure buildup methods and low pore pressure in the radius-of-investigation method, the heterogeneity and lamination of rock can be identified by the change of the permeability difference between the oscillating pulse and downstream pressure buildup methods and the radius-of-investigation method. The contribution of highly permeable parts of the rock becomes more important in the oscillating pulse and downstream pressure buildup methods than in the radius-ofinvestigation method as pore pressure increases. If microcracks exist in the rock, the permeability difference between the oscillating pulse and downstream pressure buildup methods and the radius-of-investigation method is higher than those caused by heterogeneity and lamination because the increase in the microcrack permeability is more significant than that in matrix permeability when pore pressure increases. It should be noted that heterogeneity and lamination can be coupled with microcracks in many rocks, and make the decoupling of the effects of heterogeneity and lamination from microcracks very difficult. Therefore, further work needs to be done to quantify or identify the causes of differences through advanced imaging technology.

\section{Summary and Conclusions}

The nature of tight rock makes their permeability and porosity measurements highly uncertain. In this study permeability was measured with three methods. The results from the three methods help quantify measurement and modeling uncertainties associated with the permeability and the porosity that contribute to flow. Considering the disadvantages of steady-state measurement, namely that it is being time consuming and expensive, this study has value by providing three methods of permeability measurements that are faster and inexpensive. The comparison also indicates possible presence of heterogeneities laminations and microfractures that may impact the reliable measurement of basic properties in tight rocks. The authors thus conclude:

1. The radius-of-investigation method has been developed to measure tight rock permeability. Its results agree with those of the other two methods. 
2. The results from the three permeability measurement methods show reasonable agreement and provide the range of uncertainty in permeability data using unsteady-state methods. A more reliable permeability gives more accurate reservoir modeling, production forecast, and reserve evaluation, which are important to oil and gas industry.

3. The differences among the results are useful for indicating the heterogeneities. Identification of the heterogeneity and/or microcracks using imaging technologies, such as CT, NMR, and X-ray, is one of the directions for future work.

4. It is recommended to measure porosity independently to identify significant deviations from assumption of interdependency of the porosity and permeability in methods such as the oscillating pulse method.

\section{Acknowledgment}

The authors are grateful to the Petroleum Engineering Department at the University of North Dakota. This research is supported in part by the U.S. Department of Energy (DOE) under award number DE-FC26-08NT0005643 and North Dakota EPSCoR Program under award number EPS-0814442. The authors would like to thank the Wilson M. Laird Core and Sample Library and the North Dakota Geological Survey for providing core samples for this study.

\section{NOMENCLATURE}

$A$

$\mathrm{c}_{\mathrm{S}}$

$c_{g}$

$c_{t}$

$\mathrm{D}$

$k$

$L$

$M$

$m(p)$ area of the cross section of the core plug

formation compressibility

gas isothermal compressibility

total compressibility

diameter of core

permeability

length of core

molecular weight

gas pseudopressure 


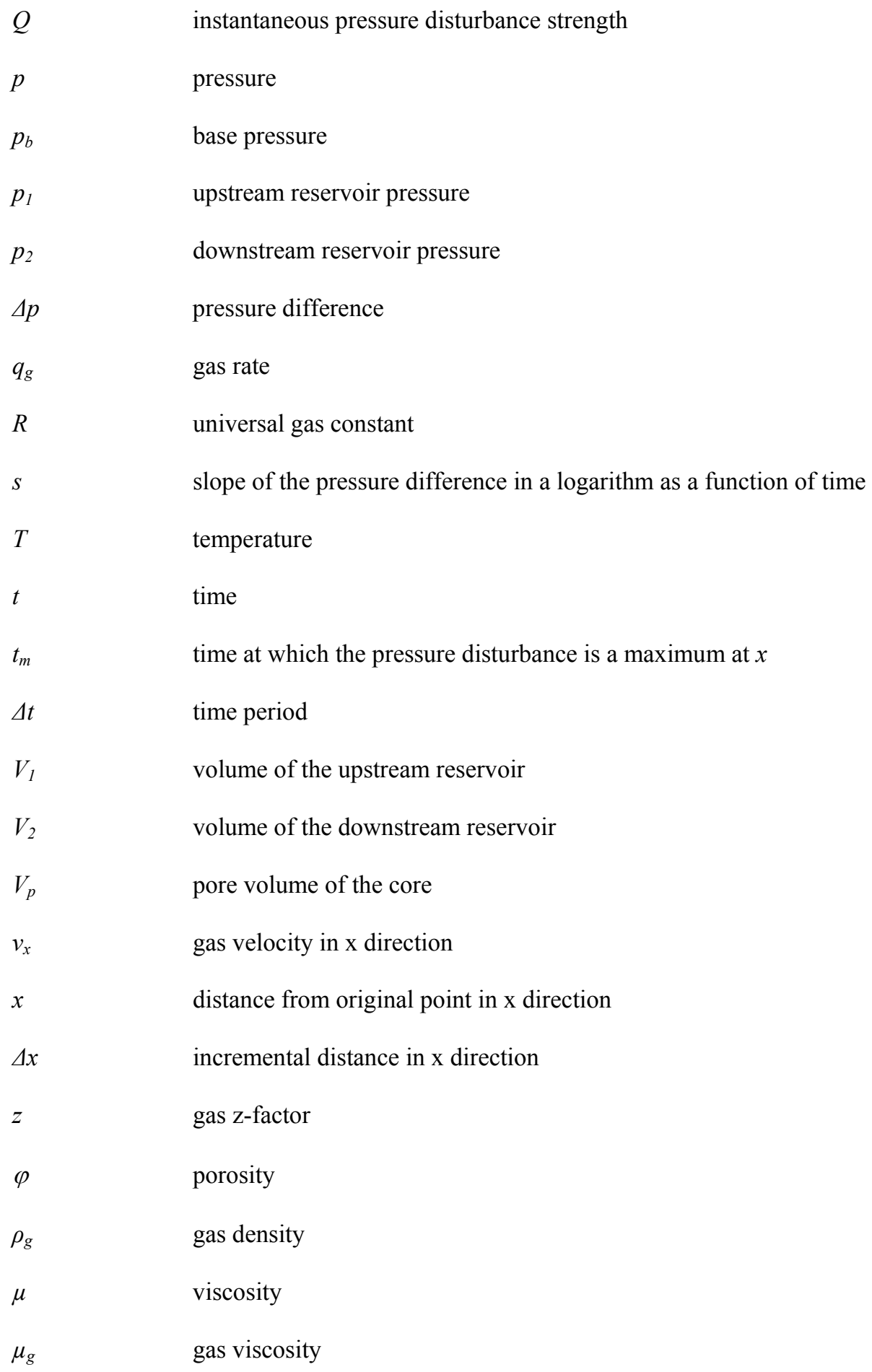

\section{REFERENCES}

Al-Hussainy, R., Ramey, H.J., and Crawford, P.B. 1966. The Flow of Real Gases through Porous Media. Journal of 
Petroleum Technology, p. 624-636.

Bertoncello, A. and Honarpour, M.M., 2013. Standards for Characterization of Rock Properties in Unconventional Reservoirs: Fluid Flow Mechanism, Quality Control, and Uncertainties. SPE 166470-MS, SPE Annual Technical Conference and Exhibition, 30 September-2 October, New Orleans, Louisiana, USA

Brace, W. F., Walsh, J. B., and Frangos, W. T. 1968. Permeability of Granite under High Pressure. Journal of Geophysical Research, p. 2225-2236.

Boitnott, G.N., 1997. Use of Complex Pore Pressure Transients to Measure Permeability of Rocks. SPE 38717-MS, SPE Annual Technical Conference and Exhibition, 5-8 October, San Antonio, Texas.

Carslaw, H. S., and Jaeger, J. C. 1959. Conduction of Heat in Solids, second edition. Oxford at Clarendon Press, P50.

Cui, X., Bustin, A.M.M., Bustin, R.M., 2009. Measurements of gas permeability and diffusivity of tight reservoir rocks: different approaches and their applications. Geofluids 9, P208-223.

Darcy, H. 1856. Les fontaines publiques de la ville de Dijon. Paris: V. Dalmont.

Dicker, A. I., and Smits, R. M. 1988. A Practical Approach for Determining Permeability from Laboratory PressurePulse Decay Measurements. SPE 17578, p. 285-292.

Hsieh, P. A., Tracy, J. V., Neuzil, C. E., Bredehoeft, J. D., and Silliman, S. E. 1981. A transient laboratory method for determining the hydraulic properties of 'tight' rocks-I. Theory. International Journal of Rock Mechanics and Mining Sciences \& Geomechanics Abstracts, 18, p. 245-252.

Jones, S. C. 1997. A Technique for Faster Pulse-Decay permeability Measurements in Tight Rocks. SPE 28450.

Kranz, R. L., Saltzman, J. S., and Blacic, J. D. 1990. Hydraulic diffusivity measurements on laboratory rock samples using an oscillating pore pressure method. International Journal of Rock Mechanics and Mining Sciences \& Geomechanics Abstracts 27, p. 345-352.

Lee, w. J. 1982. Well Testing. SPE Text book Series V1, p.13-15.

Metwally, Y. M. and Sondergeld, C. H. 2011. Measuring low permeabilities of gas-sands and shales using a pressure transmission technique. International Journal of Rock Mechanics \& Mining Sciences, p. 1135-1144.

Passey, Q.R., Bohacs, K.M., Esch, W.L., Klimentidis, R., and Sinha, S. 2010. From Oil-Prone Source Rock to GasProducing Shale Reservoir-Geologic and Petrophysical Characterization of Unconventional Shale-Gas Reservoirs. SPE 131350 presented at the CPS/SPE International Oil\& Gas Conference and Exhibition, 
Beijing, China, 8-10 June, 2010.

Sinha, S., Braun, E.M., Passey, Q.R., Leonardi, S.A., Wood, A.C., Zirkle, T., Boros, J.A., Kudva, R.A., 2012. Advances in Measurement Standards and Flow Properties Measurements for Tight Rocks such as Shales. SPE 152257-MS, SPE/EAGE European Unconventional Resources Conference and Exhibition, 20-22 March, Vienna, Austria

Tinni, A., Fathi, E., Agarwal, R., Sondergeld, C., Akkutlu, Y., and Rai, C. 2012. Shale Permeability Measurements on Plugs and Crushed Samples. SPE 162235, Canadian Unconventional Resources Conference, 30 October-1 November 2012, Calgary, Alberta, Canada.

\section{Appendix A: Oscillating Pulse Method}

Kranz et al. (1990) derived an analytical solution of the diffusion equation for 1-D flow along a finite sample excited by a pore pressure oscillation. The problem consists on finding $P(x, t)$ such that

$$
\frac{\partial P}{\partial t}=D \frac{\partial^{2} P}{\partial x^{2}}(D>0)(0<x<L)
$$

where $D$ is the coefficient of diffusion, with two boundary conditions:

(1) At $x=0, P(0, t)=A e^{i \omega t}$,

(2) At $x=L, \frac{\partial P}{\partial t}+\lambda \frac{\partial P}{\partial x}=0(\lambda>0)$

where $\omega$ is the angular frequency, $\lambda=\frac{k A}{\mu c_{g} V_{2}}$. Note $k$ is the permeability, $A$ is the area of cross-section, $V_{2}$ is the volume of the downstream reservoir, and $\mu$ and $c_{g}$ are the viscosity and compressibility of the gas, respectively.

The periodic solution as a function of distance $x$ from the upstream and time $t$ is

$$
P(x, t)=\frac{A\left\{[i \omega-\lambda(1+i) N] e^{i \omega t+(1+i) N(x-L)}-[i \omega+\lambda(1+i) N] e^{i \omega t-(1+i) N(x-L)}\right\}}{[i \omega-\lambda(1+i) N] e^{-(1+i) \mathrm{NL}}-[i \omega+\lambda(1+i) N] e^{(1+i) \mathrm{NL}}}
$$


where $N=\sqrt{\frac{\omega}{2 D}}$. Thus, at $x=L, P(x, t)$ reduces to:

$$
P(\mathrm{~L}, t)=\frac{A e^{i \omega t}[-2 \lambda(1+i) N]}{[i \omega-\lambda(1+i) N] e^{-(1+i) \mathrm{NL}}-[i \omega+\lambda(1+i) N] e^{(1+i) \mathrm{NL}}}
$$

By comparing $P(L, t)$ with $P(0, t)$, we have

$\frac{P(\mathrm{~L}, t)}{P(0, t)}=\frac{-2 \lambda(1+i) N}{[i \omega-\lambda(1+i) N] e^{-(1+i) \mathrm{NL}}-[i \omega+\lambda(1+i) N] e^{(1+i) \mathrm{NL}}}$

The ratios of $P(L, t)$ to $P(0, t)$ is a function of $\lambda$ and $N$. With two dimensionless variables $\alpha=\frac{N \lambda}{\omega}$, and $\gamma=N L$, the measurable amplitude ratios $R$ and phase difference are expressed in terms of $\alpha$ and $\gamma$ :

$$
\begin{aligned}
& R^{2}=\frac{4 \alpha^{2}}{\left(2 \alpha^{2}+1\right) \cosh 2 \gamma+\left(2 \alpha^{2}-1\right) \cos 2 \gamma+2 \alpha(\sinh 2 \gamma-\sin 2 \gamma)} \\
& \delta=\arctan \left[\frac{\tanh (2 \alpha \tan \gamma+1)+\tan \gamma}{\tan \gamma-\tanh \gamma+2 \alpha}\right]
\end{aligned}
$$

After measuring $R$ and $\delta$ from experimental measurement, we can calculate $\alpha$ and $\gamma$ from Equations (A-5) and (A6). Then permeability can be determined with Equation (A-7)

$$
k=\frac{\mu c_{g} V_{2}}{A} \lambda=\frac{\mu c_{g} V_{2}}{A}\left(\frac{\alpha \omega L}{\gamma}\right)
$$

\section{Appendix B: Downstream Pressure Buildup Method}

Hsieh et al. (1981) and Dicker and Smits (1988) gave the exact solution to Equation (1) for the pressure in the downstream reservoir, which is: 


$$
\begin{aligned}
& \frac{m\left[p_{2}(t)\right]-m\left[p_{2}(0)\right]}{m\left[p_{1}(0)\right]-m\left[p_{2}(0)\right]} \\
& =\frac{b}{a+b+a b}+2 \sum_{m=1}^{\infty} \frac{e^{-t_{D} \theta_{m}{ }^{2}}\left(a b^{2}-b \theta_{m}{ }^{2}\right)}{\left[\theta_{m}{ }^{4}+\theta_{m}{ }^{2}\left(a+a^{2}+b+b^{2}\right)+a b(a+b+a b)\right] \cos \theta_{m}}
\end{aligned}
$$

where $\theta_{m}$ can be calculated from the following equation

$$
\tan \theta=\frac{(a+b) \theta}{\theta^{2}-a b}
$$

where $a$ is the ratio of the sample pore volume $\left(V_{p}\right)$ over the upstream reservoir volume $\left(V_{l}\right)$; and $b$ is the ratio of the sample pore volume over the downstream reservoir volume $\left(V_{2}\right),\left(a=\frac{V_{p}}{V_{1}}, b=\frac{V_{p}}{V_{2}}\right)$.

In Equation (B-1) the dimensionless time, $t_{D}$, is defined as:

$$
t_{D}=\frac{k t}{\varphi \mu c_{t} L^{2}}
$$

By careful observation it is clear that $u z$ can be treated as a constant in the range of pressure less than $2000 \mathrm{psi}$,

which is the pressure conditions used in this study. According to

$$
m(p)=\int_{p_{b}}^{p} \frac{2 p}{\mu z} d p
$$
, the left-hand side (LHS) of Equation (B-1) can be written as

$$
\frac{m\left[p_{2}(t)\right]-m\left[p_{2}(0)\right]}{m\left[p_{1}(0)\right]-m\left[p_{2}(0)\right]}=\frac{\int_{p_{2}(0)}^{p_{2}(t)} \frac{2 p}{u z} d p-\int_{p_{2}(0)}^{p_{2}(0) 2 p} \frac{u p}{u z} d p}{\int_{p_{2}(0)}^{p_{1}(0)} \frac{2 p}{u z} d p-\int_{p_{2}(0)}^{p_{2}(0) 2 p} \frac{2 p}{u z} d p}=\frac{p_{2}^{2}(t)-p_{2}{ }^{2}(0)}{p_{1}^{2}(0)-p_{2}^{2}(0)}
$$

Next, the RHS of Equation (B-1) is simplified. The upstream pressure $p_{1}$ is invariant throughout the test, which implies that the upstream volume $V_{l}$ leads to infinity, so the ratio of the sample pore volume to the upstream volume ( $a=\frac{V_{p}}{V_{l}}$ ) can be considered as zero. Substituting $a$ as zero and Equation (B-4) into Equation (B-1), we obtain: 
$\frac{p_{2}^{2}(t)-p_{2}^{2}(0)}{p_{1}^{2}(0)-p_{2}^{2}(0)}=1+2 \sum_{m=1}^{\infty} \frac{e^{-t_{D} \theta_{m}^{2}}\left(-b \theta_{m}^{2}\right)}{\left[\theta_{m}^{4}+\theta_{m}^{2}\left(b+b^{2}\right)\right] \cos \theta_{m}}$

which can be written as

$\frac{p_{1}^{2}(0)-p_{2}^{2}(t)}{p_{1}^{2}(0)-p_{2}^{2}(0)}=2 \sum_{m=1}^{\infty}\left(e^{-t_{D} \theta_{m}^{2}} \cdot \frac{b \theta_{m}^{2}}{\left[\theta_{m}^{4}+\theta_{m}^{2}\left(b+b^{2}\right)\right] \cos \theta_{m}}\right)$

For $a=0$, Equation (B-2) becomes

$\tan \theta=\frac{b}{\theta}$

which can be written in the following format $\frac{\sin \theta}{\cos \theta}=\frac{b}{\theta}$ that leads to $\cos \theta=\sqrt{\frac{\theta^{2}}{\theta^{2}+b^{2}}}$. This equation contains an infinite number of solution $\theta_{m}$ and the values of the solutions increase monotonically. Thus $\cos \theta_{m}=(-1)^{m-1} \frac{\theta_{m}}{\sqrt{\theta_{m}^{2}+b^{2}}}$. Inserting this into Equation (A-5), we obtain

$\frac{p_{1}{ }^{2}(0)-p_{2}{ }^{2}(t)}{p_{1}{ }^{2}(0)-p_{2}{ }^{2}(0)}=2 \sum_{m=1}^{\infty}\left(e^{-t_{D} \theta_{m}{ }^{2}} \cdot \frac{(-1)^{m-1} b \sqrt{\theta_{m}{ }^{4}+\theta_{m}{ }^{2} b^{2}}}{\theta_{m}{ }^{4}+\theta_{m}{ }^{2}\left(b+b^{2}\right)}\right)$

Although Dicker and Smits (1988) mentioned that theoretically Equation (B-7) is single exponentially decreasing only when the upstream reservoir and the downstream reservoir have the same volume, they still indicated that in order to expedite the experiment in practical operation the volume of the upstream reservoir is usually much larger than the volume of the downstream reservoir. In this case, a single exponential equation fit very well with the downstream pressure buildup curve when the right interval was selected. Thus, Equation (B-7) was simplified to:

$$
\frac{p_{1}^{2}(0)-p_{2}^{2}(t)}{p_{1}^{2}(0)-p_{2}^{2}(0)}=e^{-t_{D} \theta_{1}^{2}} \cdot\left(2 \frac{b \sqrt{\theta_{1}^{4}+\theta_{1}^{2} b^{2}}}{\theta_{1}^{4}+\theta_{1}^{2}\left(b+b^{2}\right)}\right)
$$


Letting $\Delta p(t)=p_{1}^{2}(0)-p_{2}^{2}(t)$,

$$
\frac{\Delta p(t)}{\Delta p(0)}=\left(2 \frac{b \sqrt{\theta_{1}^{4}+\theta_{1}^{2} b^{2}}}{\theta_{1}^{4}+\theta_{1}^{2}\left(b+b^{2}\right)}\right) \cdot e^{-t_{D} \theta_{1}^{2}}
$$

which can be written as:

$$
\Delta p(t)=\left(2 \Delta p(0) \cdot \frac{b \sqrt{\theta_{1}^{4}+\theta_{1}^{2} b^{2}}}{\theta_{1}^{4}+\theta_{1}^{2}\left(b+b^{2}\right)}\right) \cdot e^{-t_{D} \theta_{1}^{2}}
$$

Taking the natural log of Equation (B-10) yields:

$$
\ln [\Delta p(t)]=\ln \left(2 \Delta p(0) \cdot \frac{b \sqrt{\theta_{1}^{4}+\theta_{1}^{2} b^{2}}}{\theta_{1}^{4}+\theta_{1}^{2}\left(b+b^{2}\right)}\right)+\left(-t_{D} \theta_{1}^{2}\right)
$$

Substituting $t_{D}$ from Equation (B-3) into Equation (B-11):

$\ln [\Delta p(t)]=\ln \left(2 \Delta p(0) \cdot \frac{b \sqrt{\theta_{1}^{4}+\theta_{1}{ }^{2} b^{2}}}{\theta_{1}^{4}+\theta_{1}{ }^{2}\left(b+b^{2}\right)}\right)-\frac{\theta_{1}^{2} k}{\varphi \mu c_{t} L^{2}} t$

Assigning $s=\frac{-\theta_{1}^{2} k}{\varphi \mu c_{t} L^{2}}$, which is the slope of the pressure difference in a logarithm as a function of time based on Equation (B-12) (Figure B-1); permeability can be easily obtained from Equation (B-13) when $s$ is fitted as in Figure B-1 .

$$
k=\frac{-\varphi \mu c_{t} L^{2}}{\theta_{1}^{2}} \cdot s
$$

Using the Taylor series of $\tan \theta, \tan \theta \approx \theta+\frac{\theta^{3}}{3}$, we can calculate $\theta_{l}$ from Equation (A-6): $\theta_{1}+\frac{\theta_{1}^{3}}{3}=\frac{b}{\theta_{1}}$, and 
$\theta_{1}^{2}=\frac{3}{2}\left(-1+\sqrt{1+\frac{4}{3} b}\right)$

Substituting Equation (B-14) into Equation (B-13:

$$
k=\frac{2 \varphi \mu c_{t} L^{2} s}{3-3 \sqrt{1+\frac{4}{3} b}}
$$

Considering that $b=\frac{V_{p}}{V_{2}}=\frac{\varphi A L}{V_{2}}$, the equation becomes:

$$
k=\frac{2 \varphi \mu c_{t} L^{2} s}{3-3 \sqrt{1+\frac{4 \varphi A L}{3 V_{2}}}}
$$

\section{Appendix C: Radius-of-Investigation Method}

According to the solution to the diffusivity Equation (1), for an instantaneous pressure disturbance in an infinite linear system (Carslaw, 1959), we have:

$m(p)=\frac{Q}{\sqrt{t}} \exp \left(\frac{-x^{2}}{4 \frac{k}{\varphi \mu c_{t}} t}\right)$

where $Q$ is a constant, which is related to the strength of the instantaneous pressure disturbance.

It is a physics problem of extreme value to find the time at which the pressure disturbance reaches its maximum. The maximum solution can be solved when the time derivative of the Equation $(\mathrm{C}-1)$ equals zero: 
$\frac{d[m(p)]}{d t}=\frac{d\left[\frac{Q}{\sqrt{t}} \exp \left(\frac{-x^{2}}{4 \frac{k}{\varphi \mu c_{t}} t}\right)\right]}{d t}=0$

which is:

$\frac{d[m(p)]}{d t}=\exp \left(\frac{-x^{2}}{4 \frac{k}{\varphi \mu c_{t}} t}\right) \frac{d\left[\frac{Q}{\sqrt{t}}\right]}{d t}+\frac{Q}{\sqrt{t}} \frac{d\left[\exp \left(\frac{-x^{2}}{4 \frac{k}{\varphi \mu c_{t}} t}\right)\right]}{d t}=0$

Simplifying the above equations lead to:

$\frac{d[m(p)]}{d t}=\left[-\frac{Q}{2 t^{\frac{3}{2}}}\right] \cdot \exp \left(\frac{-x^{2}}{4 \frac{k}{\varphi \mu c_{t}} t}\right)+\left(\frac{x^{2} Q}{4 \frac{k}{\varphi \mu c_{t}} t^{\frac{5}{2}}}\right) \cdot \exp \left(\frac{-x^{2}}{4 \frac{k}{\varphi \mu c_{t}} t}\right)=0$

Finally, Equation (C-5) is obtained as:

$\frac{d[m(p)]}{d t}=\frac{Q}{2 t^{\frac{3}{2}}} \cdot \exp \left(\frac{-x^{2}}{4 \frac{k}{\varphi \mu c_{t}} t}\right) \cdot\left[-1+\frac{x^{2}}{2 \frac{k}{\varphi \mu c_{t}} t}\right]=0$ 
Considering the initial condition at $t=0$ and $p(x, t=0)=p_{2}, t=0$ is a trivial solution to Equation (C-5). Dividing

both sides of the Equation (C-5) by $\frac{Q}{2 t^{\frac{3}{2}}} \cdot \exp \left(\frac{-x^{2}}{4 \frac{k}{\varphi \mu c_{t}} t}\right)$ yields $-1+\frac{x^{2}}{2 \frac{k}{\varphi \mu c_{t}} t}=0$.

Rearranging the equation, we get the time:

$t_{m}=t=\frac{\varphi \mu c_{t} x^{2}}{2 k}$

Expressing permeability in terms of porosity, viscosity, total compressibility, location, and time, Equation (C-6) can be written as:

$k=\frac{\varphi \mu c_{t} x^{2}}{2 t_{m}}$

Converting Equation (C-7) into U.S. field units we have:

$k=\frac{1896 \varphi \mu c_{t} x^{2}}{t_{m}}$

where permeability $k$ is in $\mathrm{mD}$, porosity $\varphi$ is dimensionless (in fraction), viscosity $\mu$ is in cp, total compressibility $c_{t}$ is in $\mathrm{psi}^{-1}$, time $t_{m}$ is in hour, and location (or distance) $x$ is in $\mathrm{ft}$.

Equations (C-7) and (C-8) are the governing equations to measure the rock permeability. They are used to calculate the permeability of any rock that meets the aforementioned assumptions and can be used for high-permeability rocks as well. The proposed method evaluates the permeability under unsteady-state flow and requires a short time period to determine the flow capacity of the low-permeability rock.

\section{Appendix D: Experimental Data}

The evolutions of upstream and downstream pressures versus time and $\ln (\Delta p)$ versus time in the permeability experiment for cores \#2 to \#6 are shown in Figures D-1 through D-10. 


\begin{tabular}{|c|c|c|c|c|c|c|c|}
\hline & unit & Core 1 & Core 2 & Core 3 & Core 4 & Core 5 & Core 6 \\
\hline$L$ & in & 2.7780 & 2.7224 & 2.7008 & 2.3882 & 2.6992 & 2.5819 \\
\hline $\mathrm{D}$ & in & 1.0311 & 1.0394 & 1.0327 & 1.0323 & 1.0291 & 1.0315 \\
\hline$\varphi$ & fraction & 0.044 & 0.045 & 0.032 & 0.035 & 0.036 & 0.054 \\
\hline $\mathrm{C}_{\mathrm{s}}$ & $1 / p s i$ & 0.000009 & 0.000009 & 0.000009 & 0.000009 & 0.000009 & 0.000009 \\
\hline $\mathrm{C}_{\mathrm{g}}$ & $1 / p s i$ & 0.000125 & 0.000125 & 0.000125 & 0.000125 & 0.000125 & 0.000125 \\
\hline $\mathrm{C}_{t}$ & $1 / p s i$ & 0.000134 & 0.000134 & 0.000134 & 0.000134 & 0.000134 & 0.000134 \\
\hline$\mu$ & $\mathrm{cp}$ & 0.0293 & 0.0293 & 0.0293 & 0.0293 & 0.0293 & 0.0293 \\
\hline$V_{2}$ & $\mathrm{ft}^{3}$ & $2.22 \mathrm{E}-05$ & $2.22 \mathrm{E}-05$ & $2.22 \mathrm{E}-05$ & $2.22 \mathrm{E}-05$ & $2.22 \mathrm{E}-05$ & $2.22 \mathrm{E}-05$ \\
\hline $\mathrm{s}$ & $\operatorname{Ln}\left(p s i^{2}\right) / h$ & -2.781108 & -1.3644 & -1.818 & -1.2528 & -2.4228 & -78.12 \\
\hline$\mu$ & $\mathrm{cp}$ & 0.0293 & 0.0293 & 0.0293 & 0.0293 & 0.0293 & 0.0293 \\
\hline$t$ & $\mathrm{~h}$ & 0.0694 & 0.28 & 0.13889 & 0.14167 & 0.05556 & 0.0035 \\
\hline $\begin{array}{c}\mathrm{k} \\
\text { (buildup) }\end{array}$ & $\mu \mathrm{D}$ & 0.1864 & 0.09 & 0.1164 & 0.0791 & 0.158 & 5.2731 \\
\hline $\begin{array}{c}\mathrm{k} \\
\text { (radius-of- } \\
\text { investigation) }\end{array}$ & $\mu \mathrm{D}$ & 0.2527 & 0.0615 & 0.0868 & 0.0728 & 0.2438 & 5.3546 \\
\hline $\begin{array}{c}\mathrm{k} \\
\text { (oscillating pulse) }\end{array}$ & $\mu \mathrm{D}$ & 0.108 & 0.046 & 0.0724 & 0.0438 & 0.11 & 2.25 \\
\hline
\end{tabular}




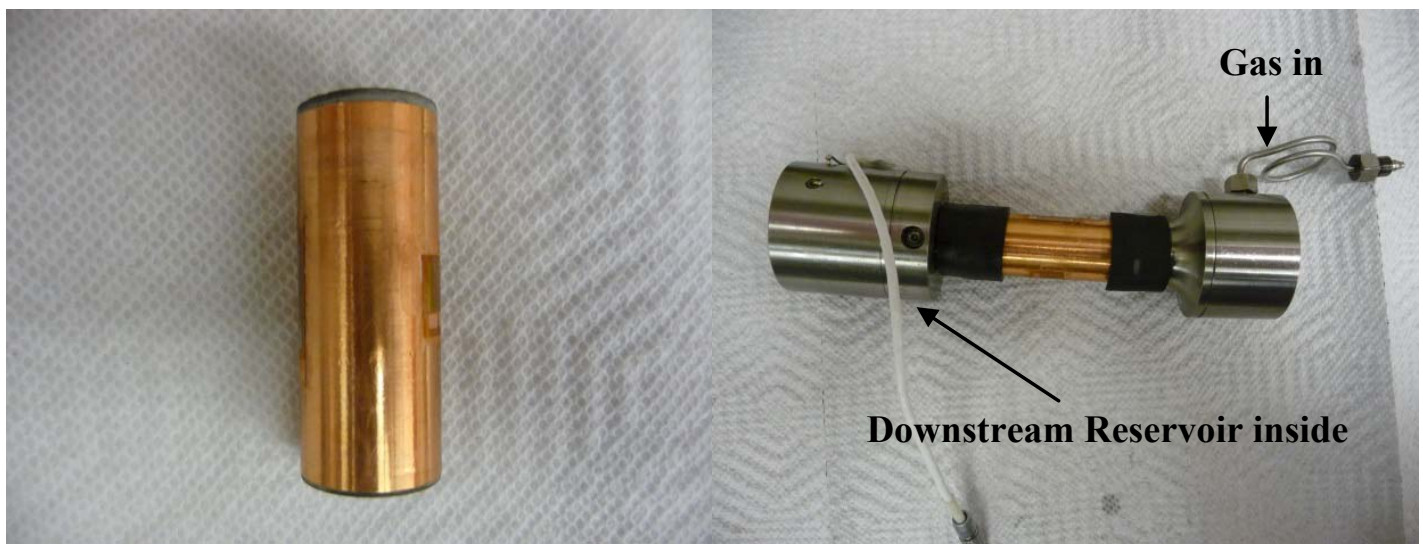

Figure 1. Core covered with copper sheeting, and assembled on End Caps for a low permeability test system 


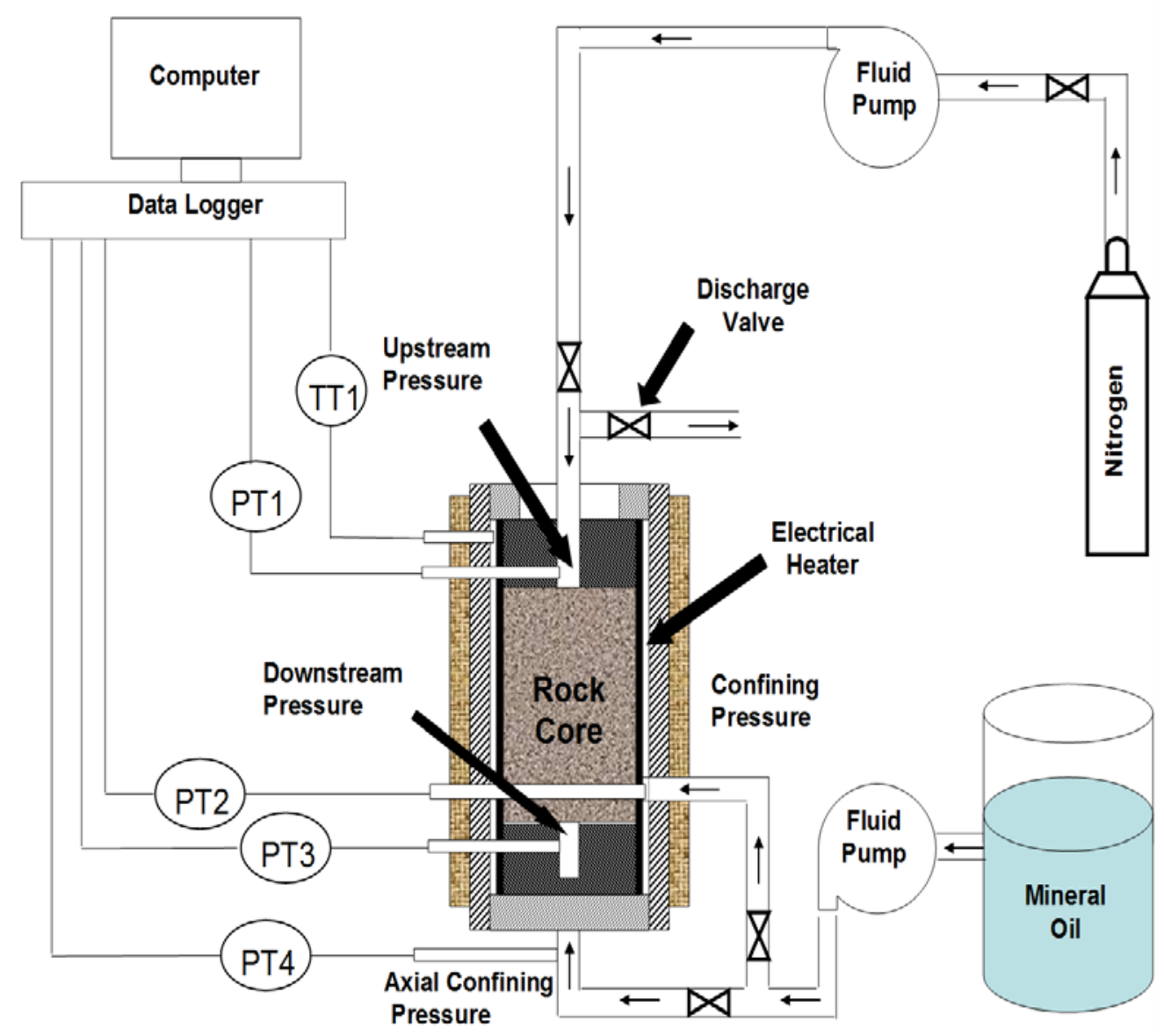

Figure 2. Schematic diagram for the experimental setup. The nitrogen vessel is the upstream extended reservoir and its pressure is measured by pressure transducer PT1; the pressure of the downstream reservoir (volume $=0.63 \mathrm{cc}$ ) is measured by PT3; the mineral oil is used for confining pressure and axial pressure systems 


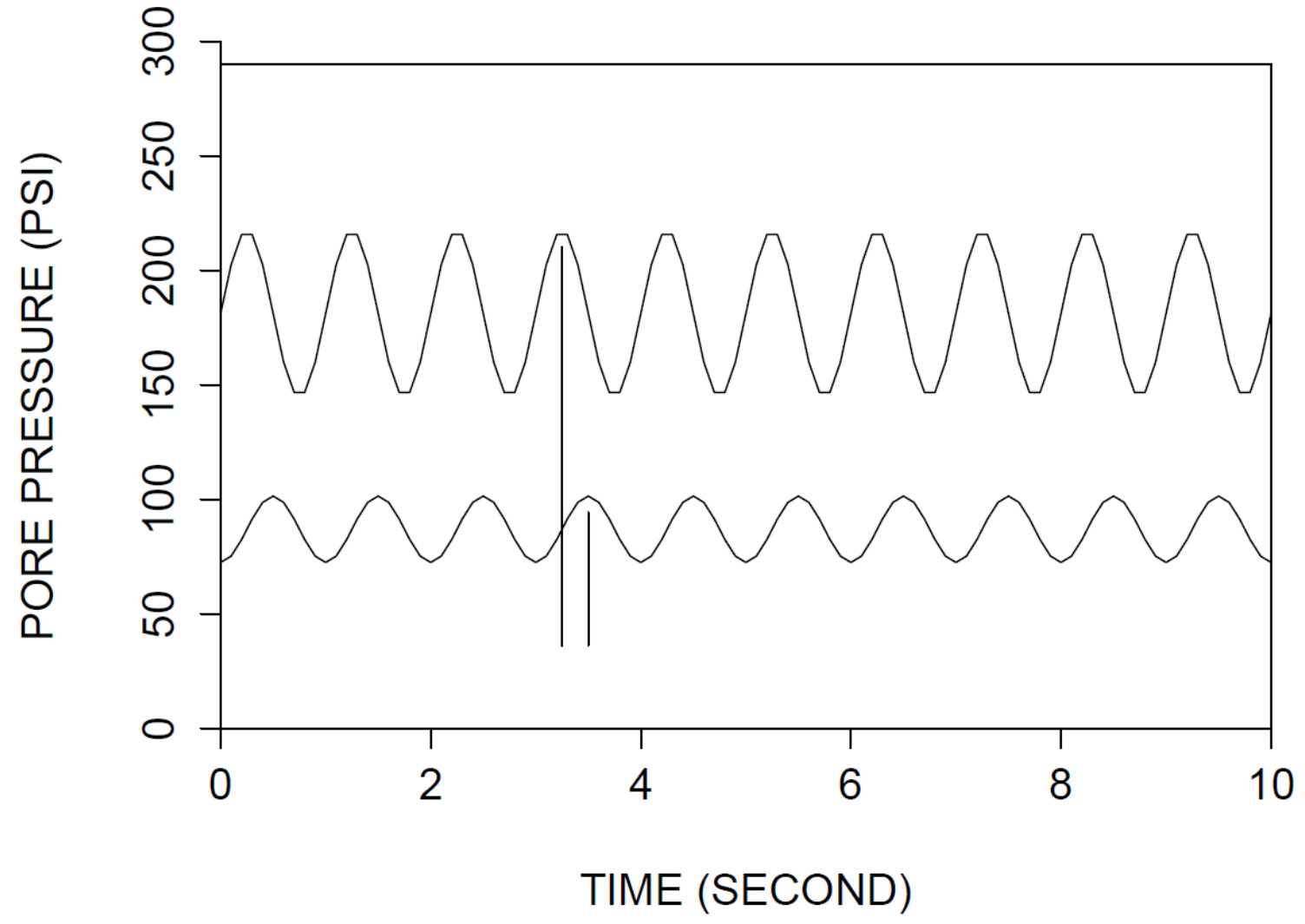

Figure 3. Illustration of the effect of the upstream input oscillation frequency on the resultant downstream amplitude and phase shift of the oscillating pulse method 


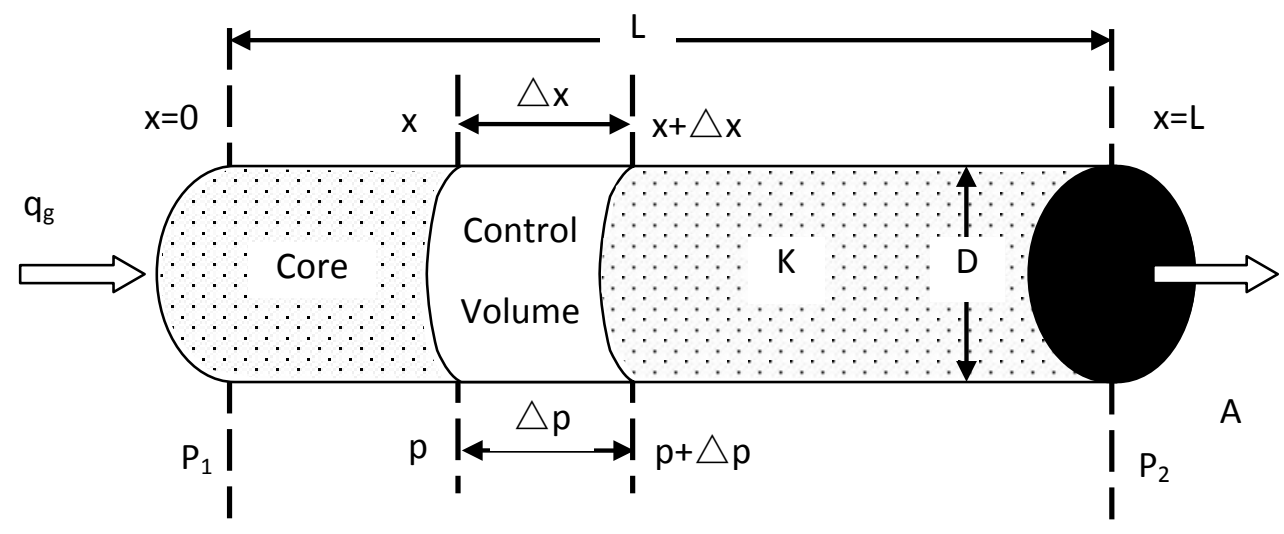

Figure 4. Schematic diagram depicting how gas flows through a core 


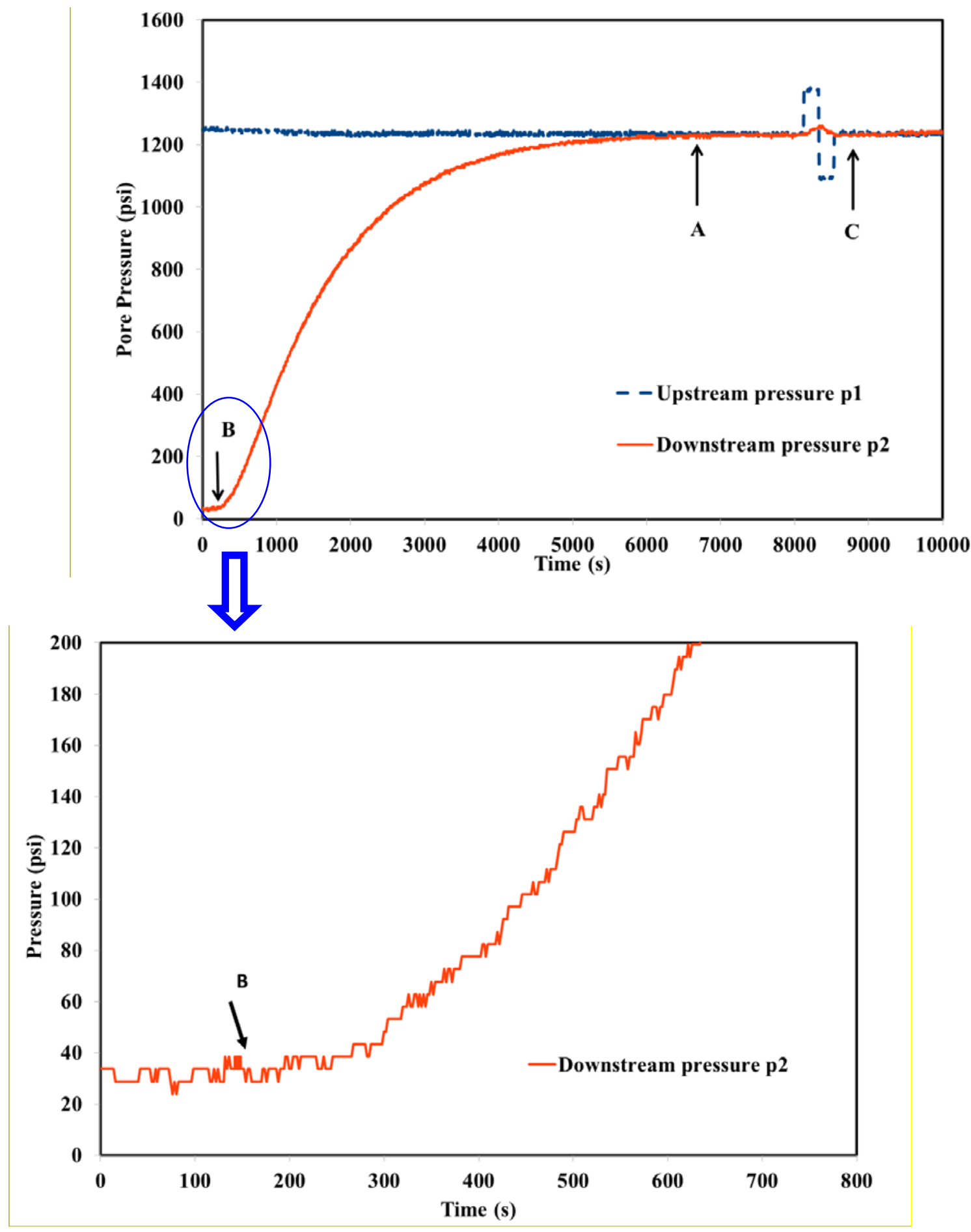

Figure 5. Changes of the upstream and downstream pressure during one experiment for Core \#1. Point "A" marks the time at which the downstream pressure buildup method stops, point "B" marks the time at which the radius-ofthe investigation method stops, and point " $\mathrm{C}$ " marks the time at which the oscillating pulse method stops 


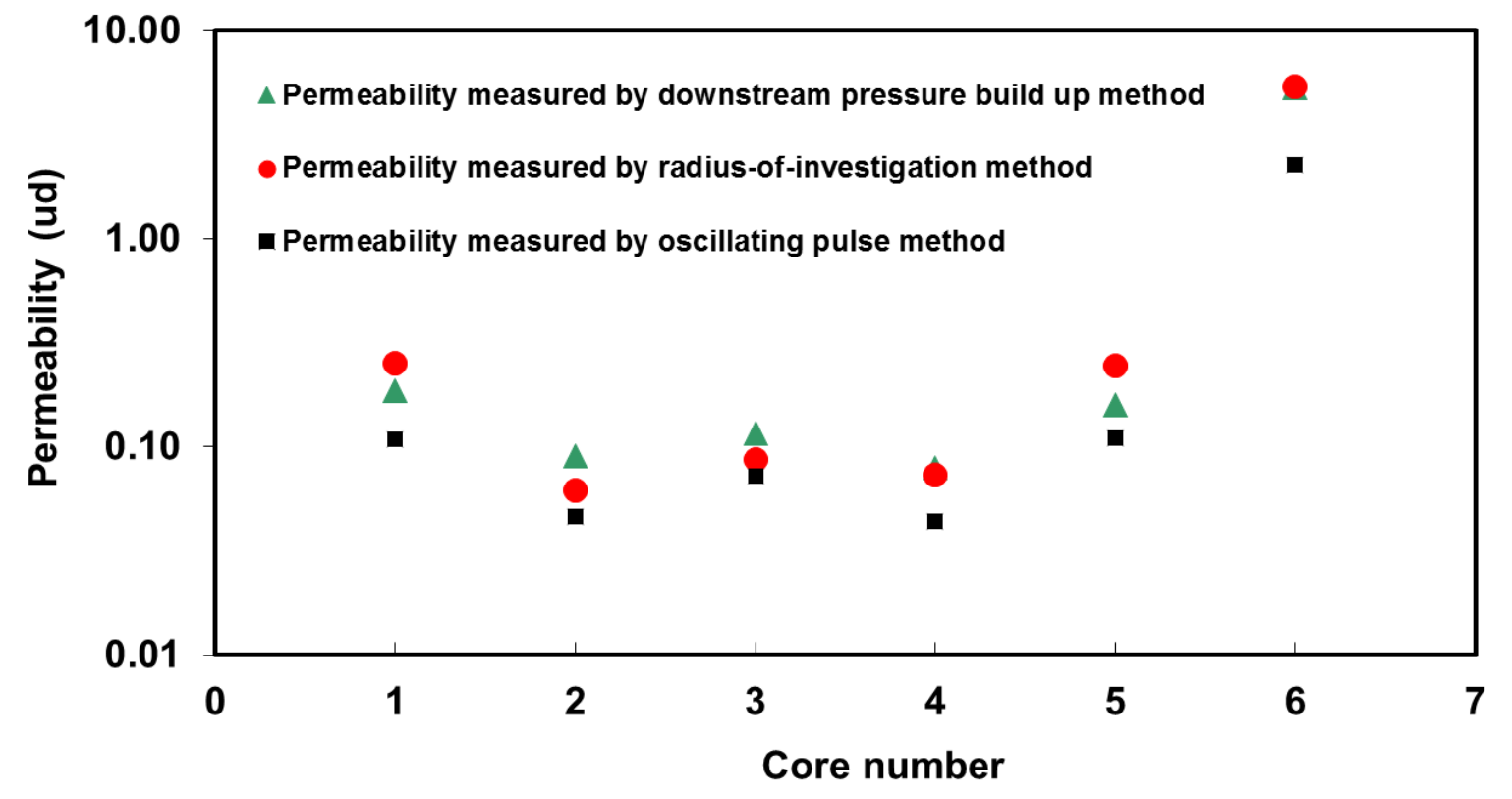

Figure 6. Comparison of permeabilities as measured by the three methods 


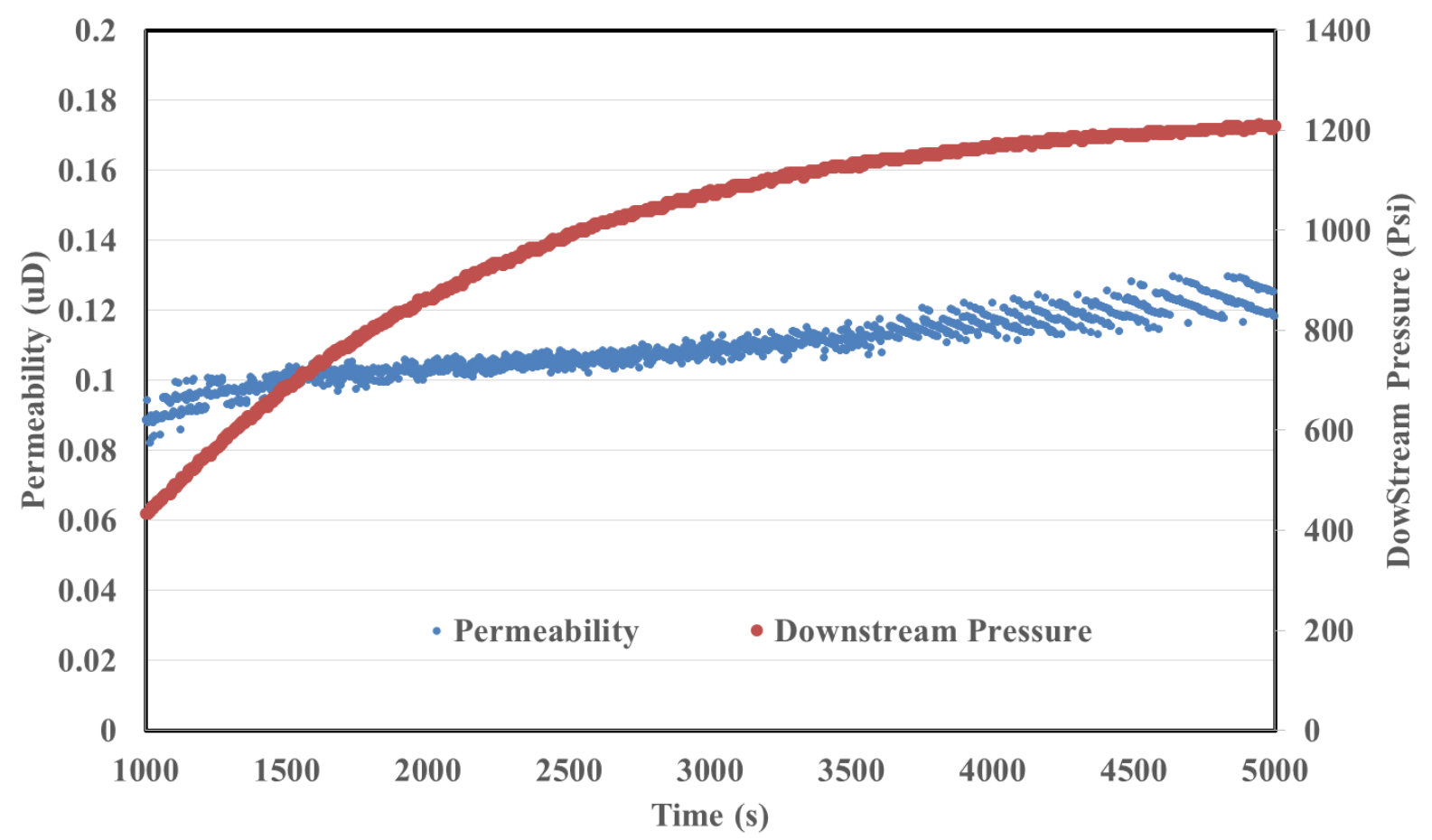

Figure 7. Downstream pressure during buildup and the calculated permeability for Core \#1 


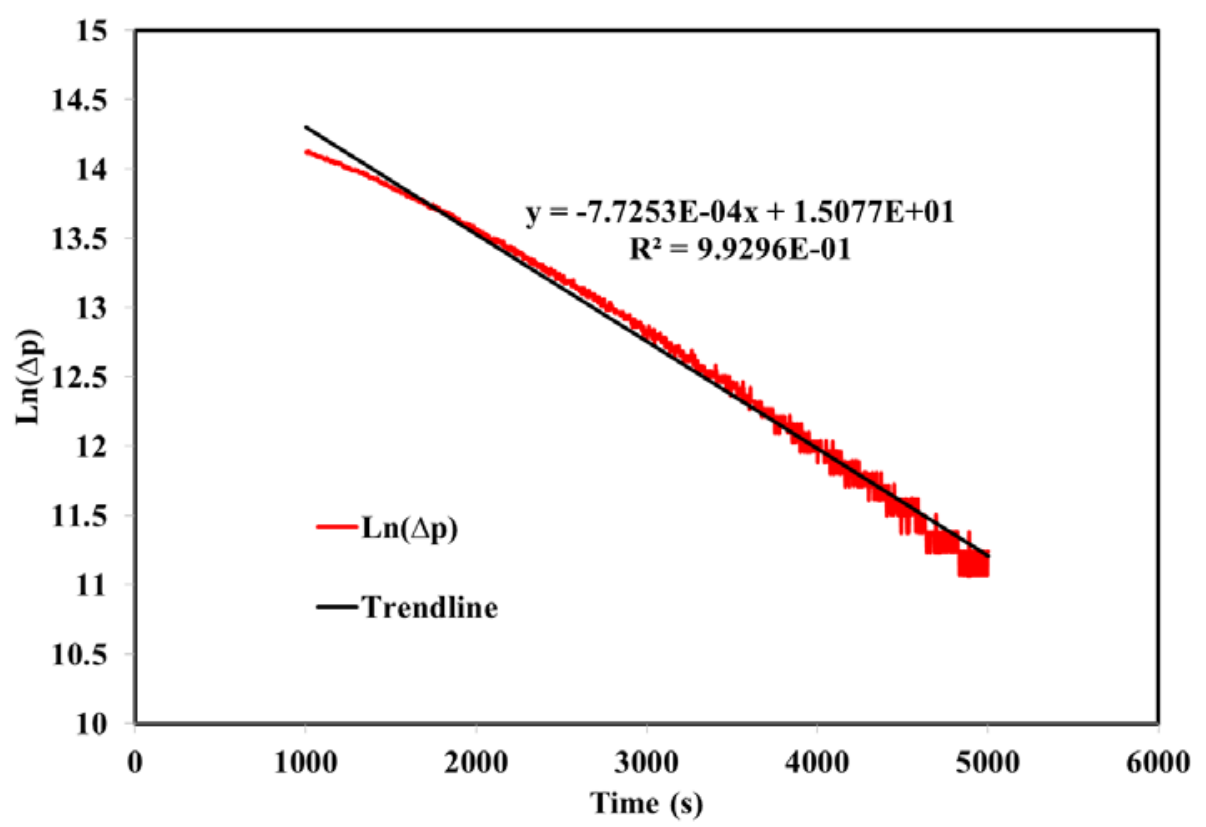

Figure B-1. $\ln (\Delta p)$ vs. time plot for Core \#1 


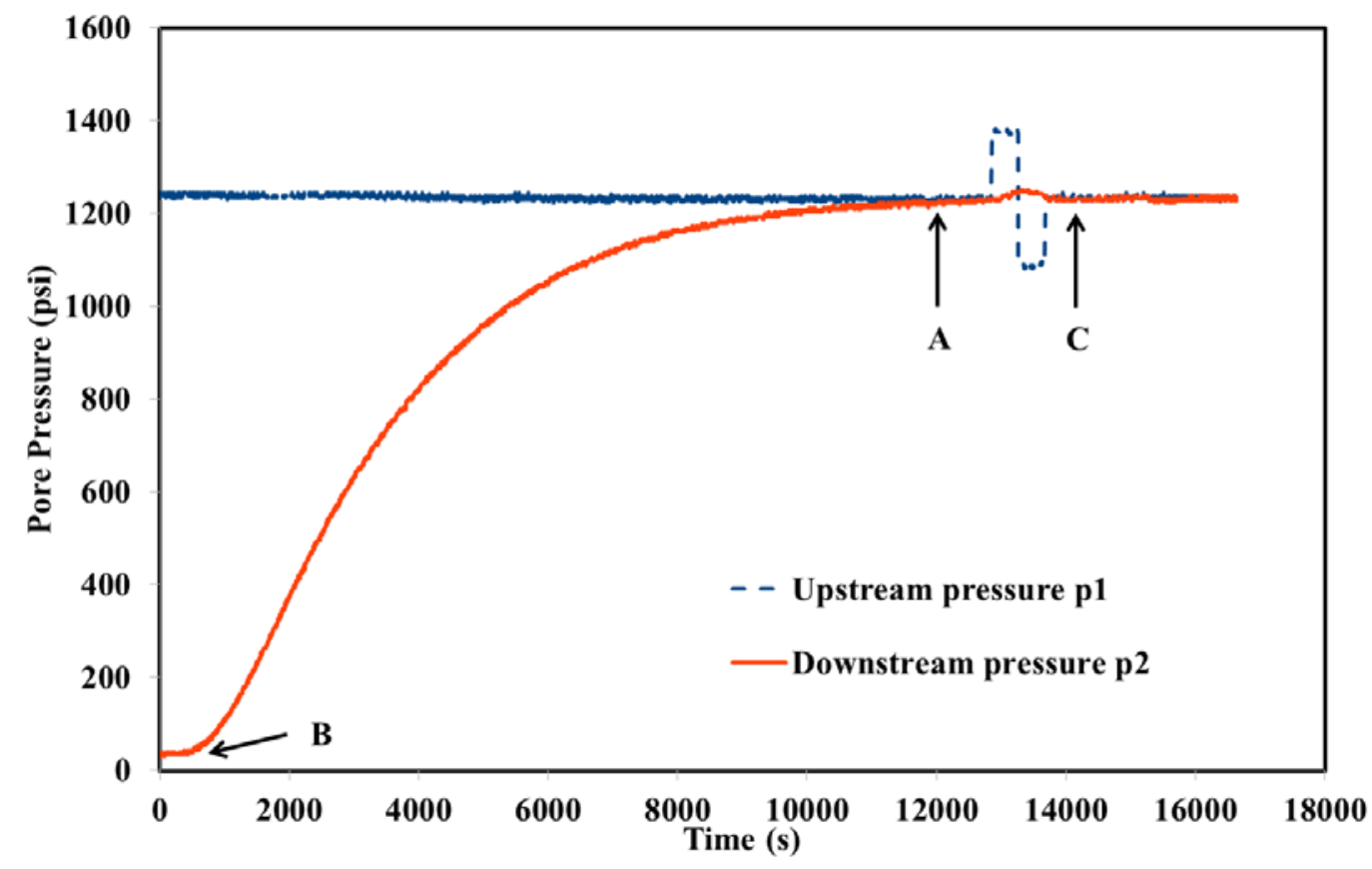

Figure D-1. Changes of the upstream and downstream pressure during one experiment for Core \#2 


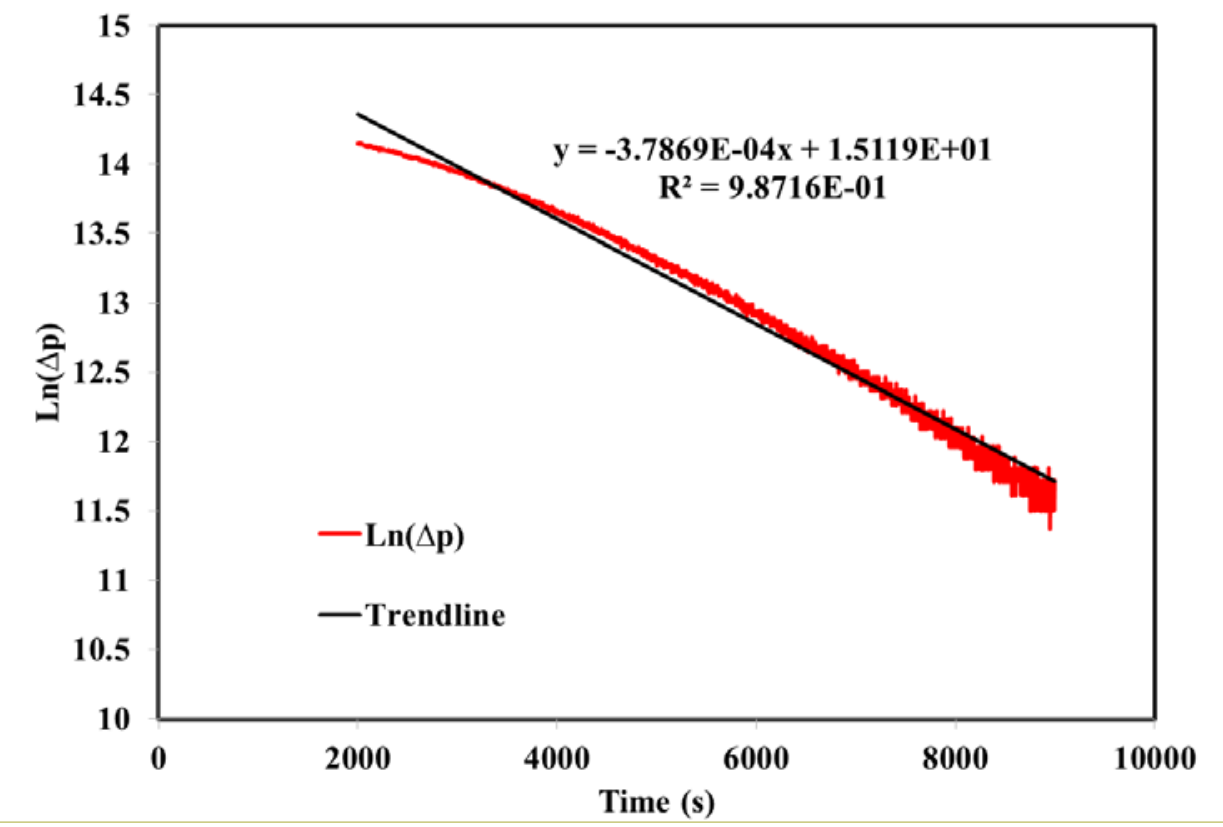

Figure D-2. $\ln (\Delta p)$ vs. time plot for Core \#2 


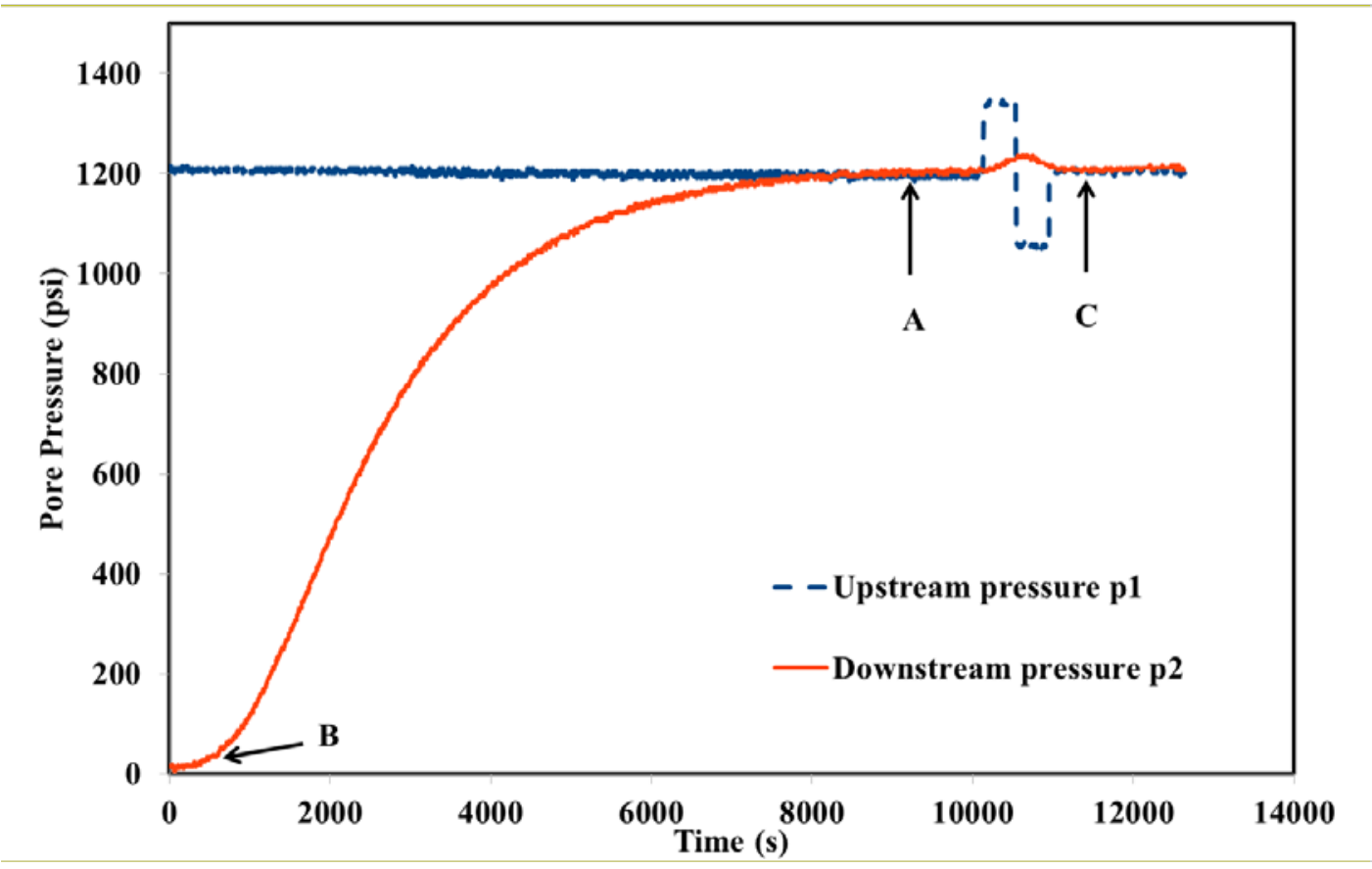

Figure D-3. Changes of the upstream and downstream pressure during one experiment for Core \#3 


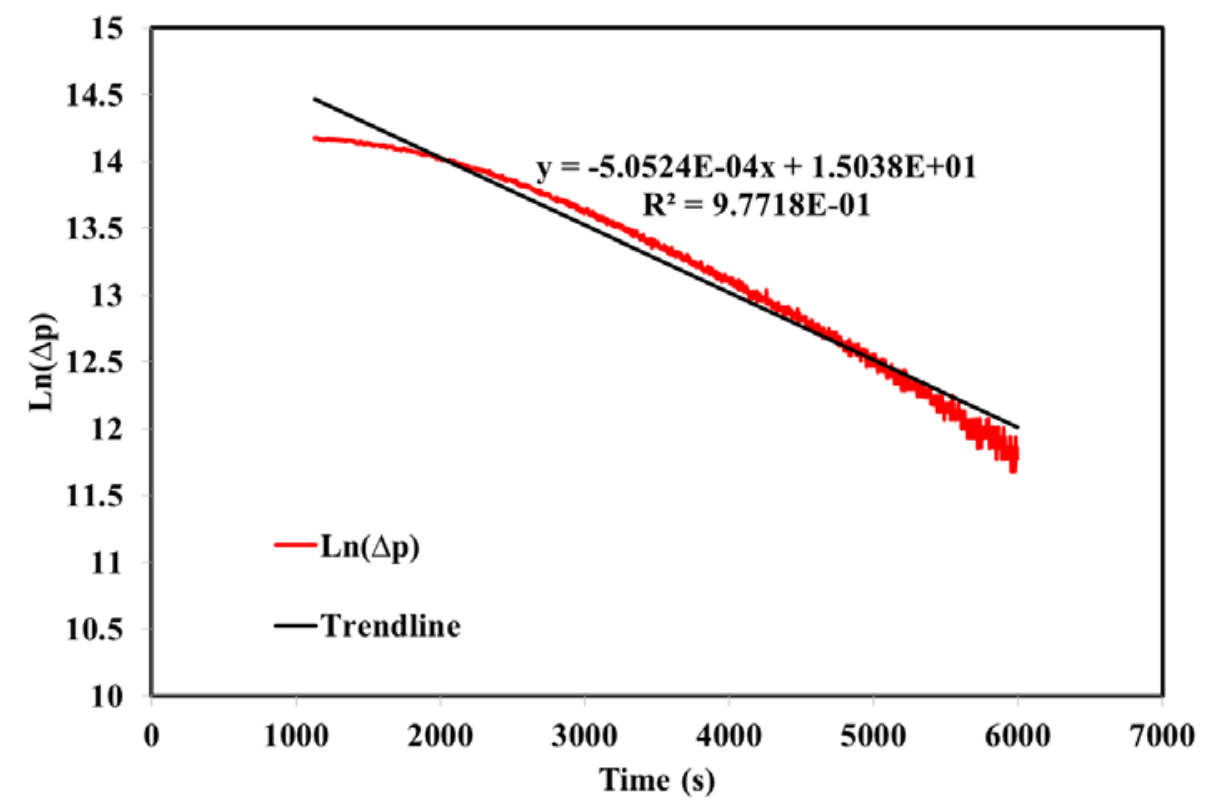

Figure D-4. $\ln (\Delta p)$ vs. time plot for Core \#3 


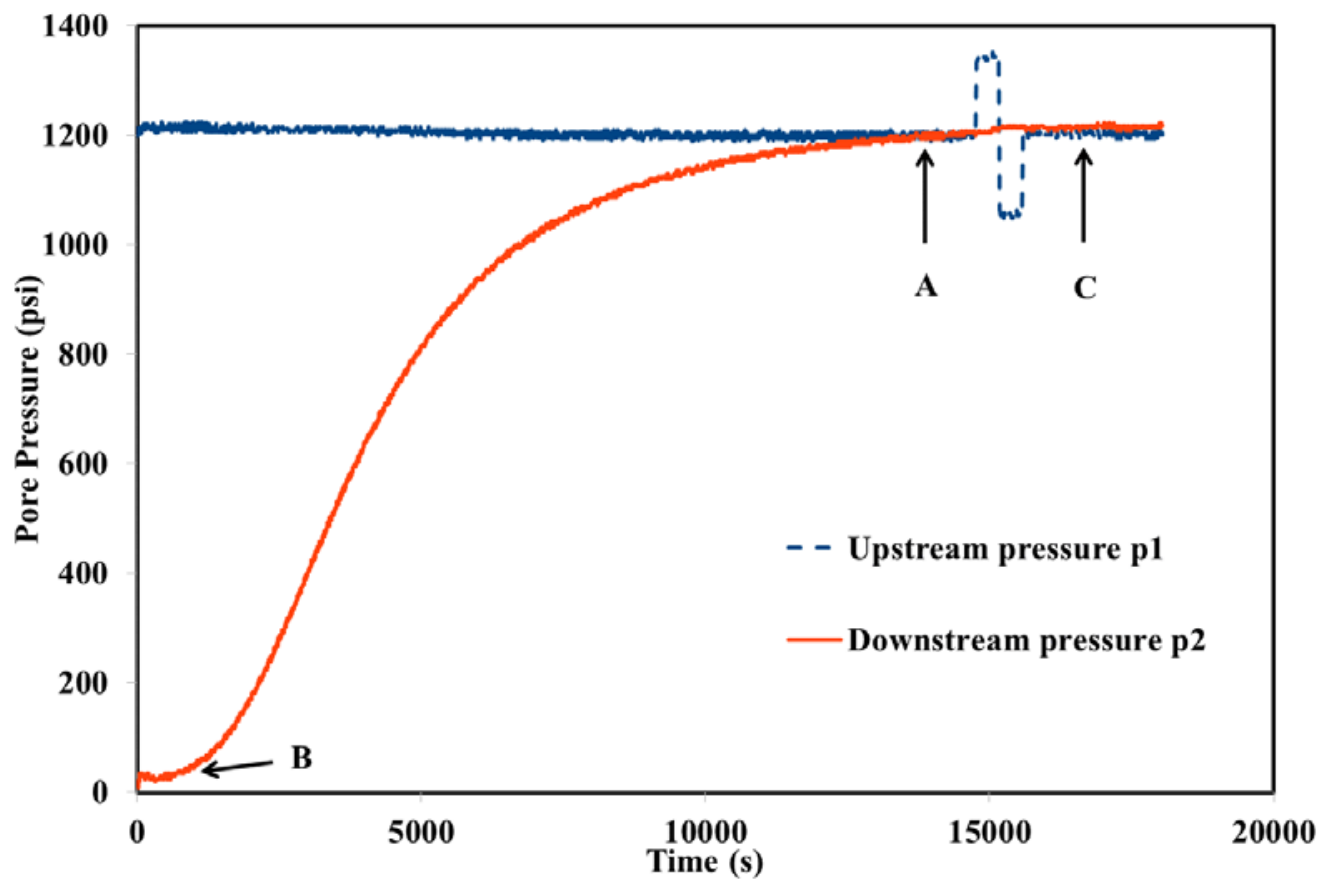

Figure D-5. Changes of the upstream and downstream pressure during one experiment for Core \#4 


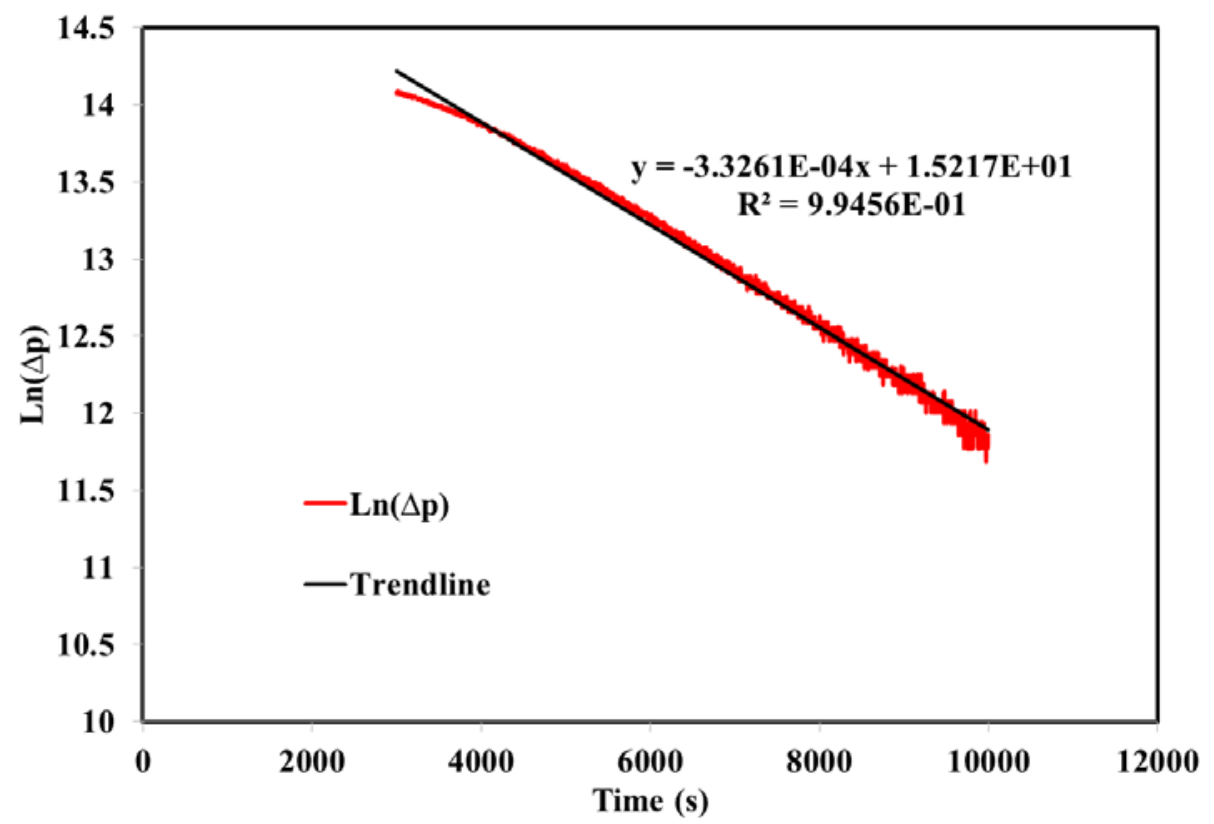

Figure D-6. $\ln (\Delta p)$ vs. time plot for Core $\# 4$ 


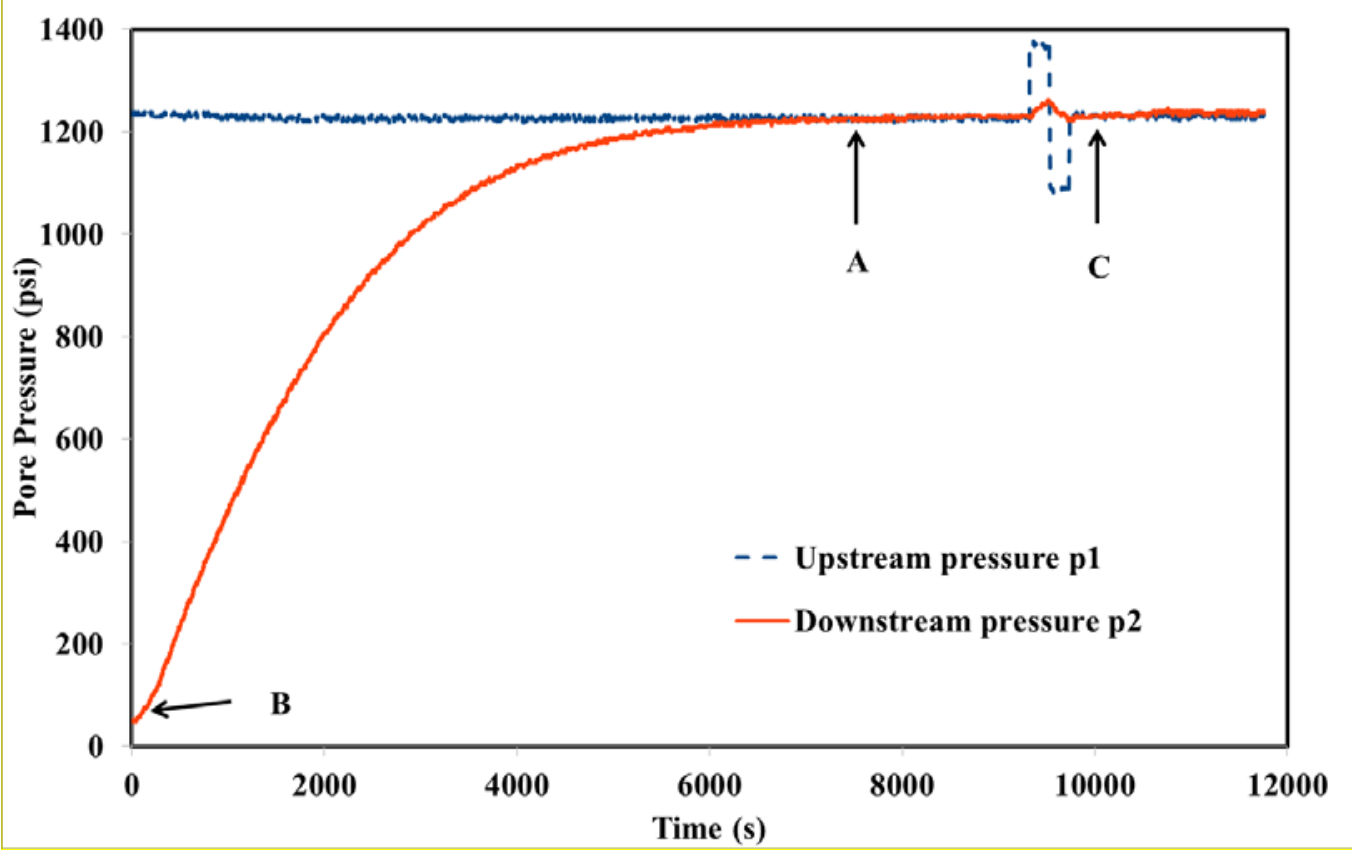

Figure D-7. Changes of the upstream and downstream pressure during one experiment for Core \#5 


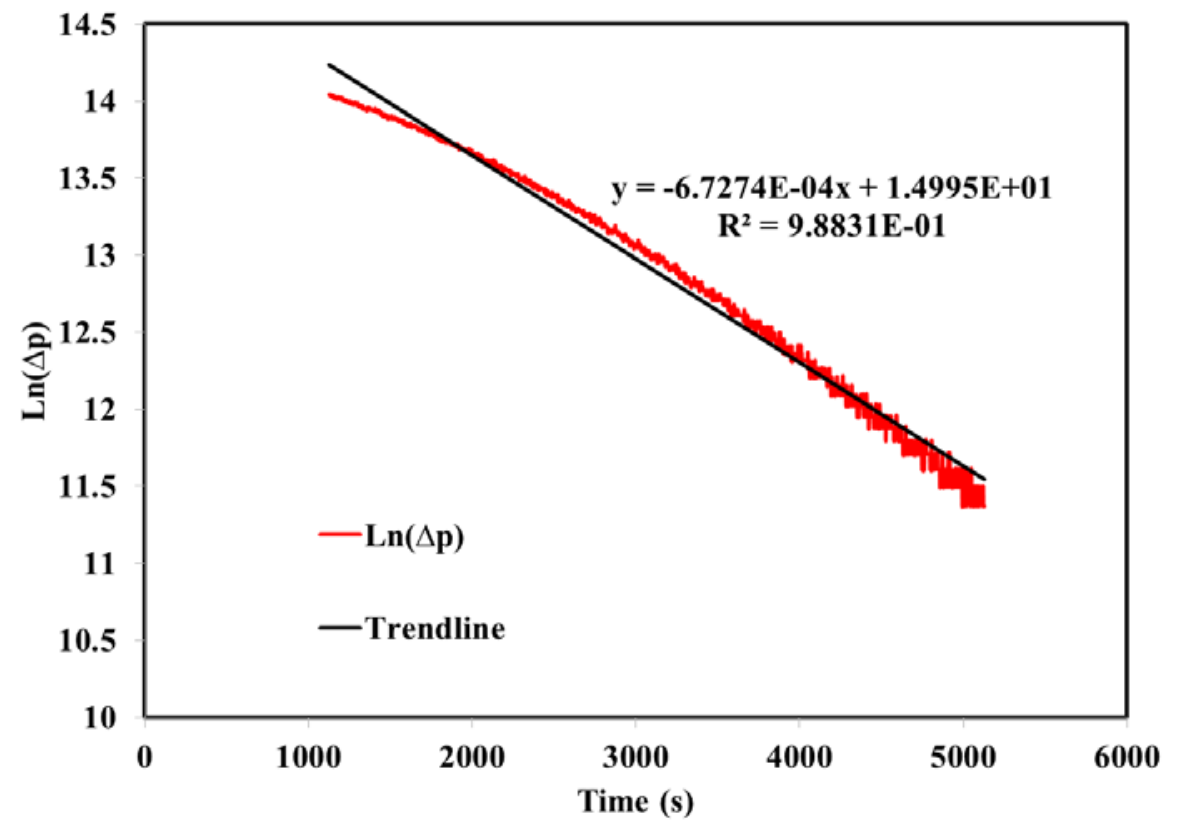

Figure D-8. $\ln (\Delta p)$ vs. time plot for Core \#5 


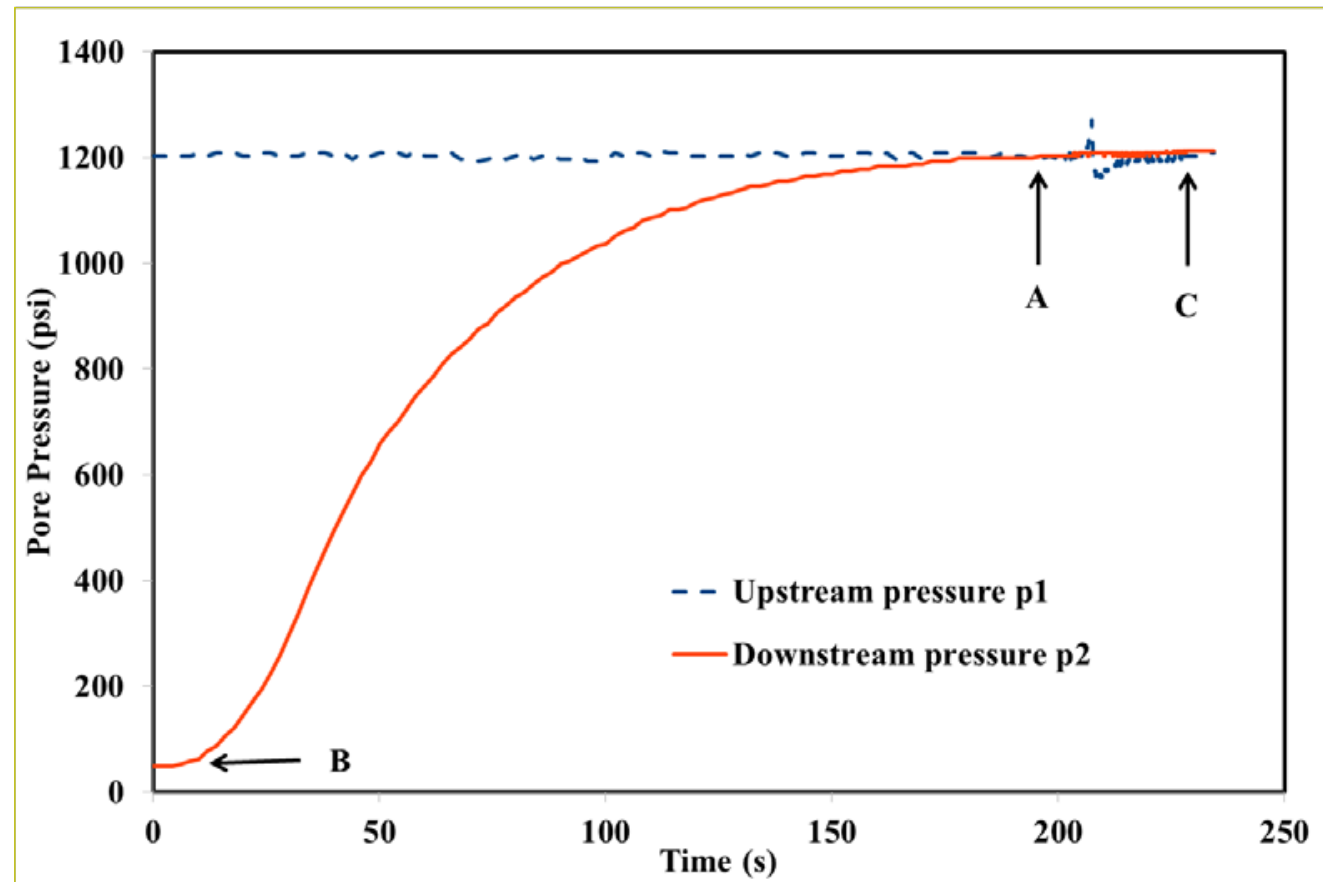

Figure D-9. Changes of the upstream and downstream pressure during one experiment for Core \#6 


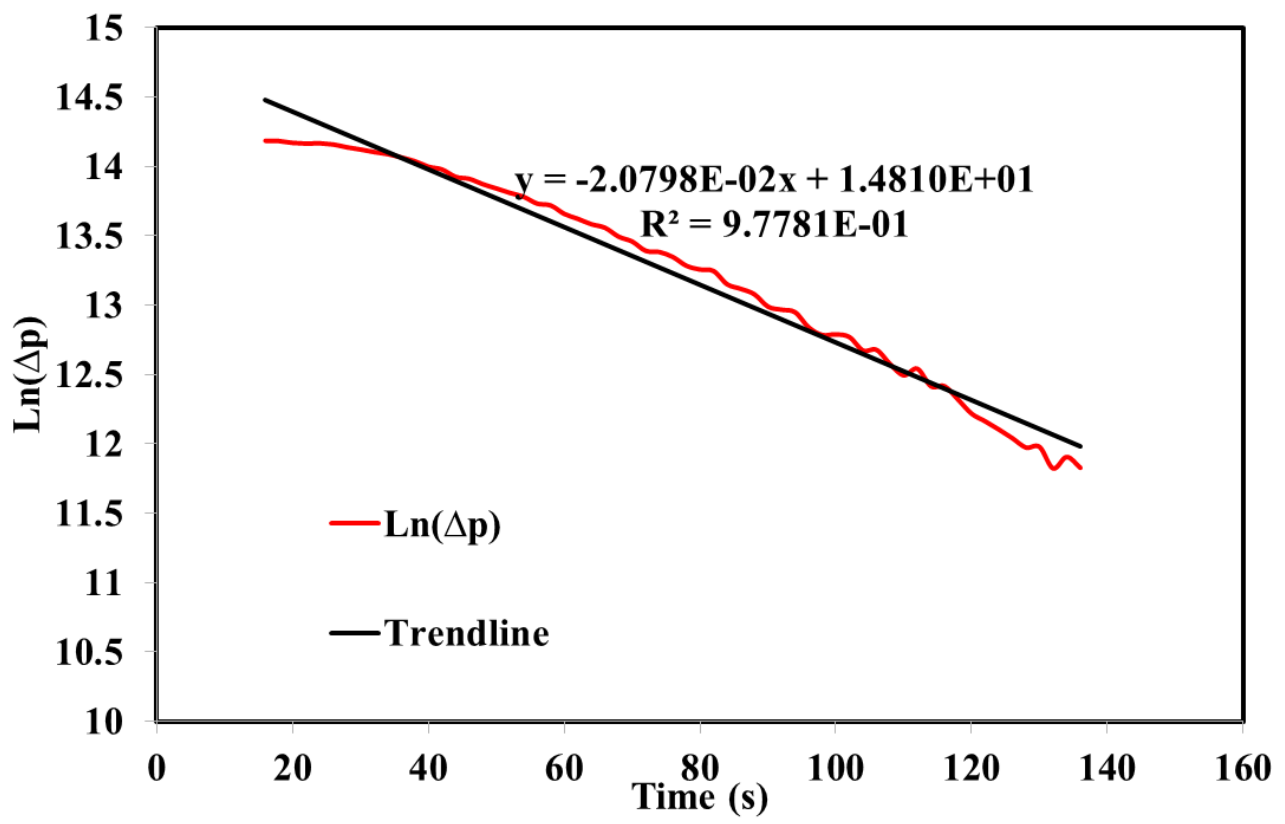

Figure D-10. $\ln (\Delta p)$ vs. time plot for Core \#6 